\title{
QUANTITATIVE UNIQUE CONTINUATION OF SOLUTIONS TO HIGHER ORDER ELLIPTIC EQUATIONS WITH SINGULAR COEFFICIENTS
}

\author{
JIUYI ZHU
}

\begin{abstract}
We investigate the quantitative unique continuation of solutions to higher order elliptic equations with singular coefficients. Quantitative unique continuation described by the vanishing order is a quantitative form of strong unique continuation property. We characterize the vanishing order of solutions for higher order elliptic equations in terms of the norms of coefficient functions in their respective Lebesgue spaces. New versions of quantitative Carleman estimates are established.
\end{abstract}

\section{INTRODUCTION}

In this paper, we study the quantitative unique continuation for higher order elliptic equations with singular lower order terms. Suppose $u$ is a non-trivial solution to

$$
\triangle^{m} u+\sum_{|\alpha|=1}^{\alpha_{0}} V_{\alpha}(x) \cdot D^{\alpha} u+V_{0}(x) u=0 \quad \text { in } \mathbb{B}_{10},
$$

where $\mathbb{B}_{10}$ is a ball centered at origin with radius 10 in $\mathbb{R}^{n}$ with $n \geq 2$, The value $\alpha_{0}$ is a positive integer. If $m$ is a positive even integer, the value $\alpha_{0} \leq\left[\frac{3 m}{2}\right]-1$. If $m$ is a positive odd integer, the value $\alpha_{0} \leq\left[\frac{3 m}{2}\right]$. Assume that $V_{\alpha}(x) \in L^{\infty}\left(\mathbb{B}_{10}\right)$ and $V_{0}(x) \in L^{s}\left(\mathbb{B}_{10}\right)$ for some positive constant $s$ to be specified later. We also normalize the solutions $u$ in (1.1) as $\|u\|_{L^{\infty}\left(\mathbb{B}_{1}\right)} \geq 1$ and $\|u\|_{L^{\infty}\left(\mathbb{B}_{10}\right)} \leq \hat{C}$.

Quantitative unique continuation described by the vanishing order characterizes how much the solution vanishes. We say vanishing order of solution at $x_{0}$ is $l$, if $l$ is the largest integer such that $D^{\alpha} u\left(x_{0}\right)=0$ for all $|\alpha|<l$, where $\alpha$ is a multi-index. It is a quantitative way to describe the strong unique continuation property. So we also call it quantitative uniqueness. Strong unique continuation property states that if a solution that vanishes of infinite order at a point vanishes identically. We know that all zeros of nontrivial solutions of second order linear equations on smooth compact Riemannian manifolds are of finite order. Especially, for classic eigenfunctions on a compact smooth Riemannian manifold $\mathcal{M}$,

$$
-\triangle_{g} \phi_{\lambda}=\lambda \phi_{\lambda} \quad \text { in } \mathcal{M} \text {. }
$$

Donnelly and Fefferman in [DF88] showed that the maximal vanishing order of $\phi_{\lambda}$ is everywhere less than $C \sqrt{\lambda}$, here $C$ only depends on the manifold $\mathcal{M}$. The vanishing order of classical eigenfunction $\phi_{\lambda}$ is sharp and its sharpness can be seen from spherical harmonics if $\mathcal{M}$ is a sphere. If the strong unique continuation property holds for the solutions and solutions do not vanish of infinite order, the vanishing order of solutions depends on the potential functions and coefficient functions appeared in the equations. It is interesting to characterize the vanishing order by the potential function $V_{0}(x)$ and coefficient functions $V_{\alpha}(x)$ in (1.1).

2010 Mathematics Subject Classification. 35J15, 35J10, 35A02.

Key words and phrases. Carleman estimates, unique continuation, higher order elliptic equations, vanishing order.

Zhu is supported in part by NSF grant DMS-1656845. 
Recently, there has been much interest in investigating the vanishing order of solutions for (1.1) in the case $m=1$, i.e. the second order elliptic equation

$$
\triangle u+V_{1}(x) \cdot \nabla u+V_{0}(x) u=0 \quad \text { in } \mathbb{B}_{10} .
$$

Kukavica in [Ku98] studied the vanishing order of solutions for Schrödinger equation

$$
\triangle u+V_{0}(x) u=0 .
$$

If $V_{0}(x) \in W^{1, \infty}$, Kukavica established that the upper bound of vanishing order is less than $C\left(1+\left\|V_{0}\right\|_{W^{1, \infty}}\right)$, where $\left\|V_{0}\right\|_{W^{1, \infty}}=\left\|V_{0}\right\|_{L^{\infty}}+\left\|\nabla V_{0}\right\|_{L^{\infty}}$. This upper bound is not sharp, which can be seen from Donnelly and Fefferman's work in the case $V_{0}(x)=\lambda$. Recently, the sharp vanishing order for solutions of $(1.3)$ is shown to be less than $C\left(1+\sqrt{\left\|V_{0}\right\|_{W^{1, \infty}}}\right)$ independently by Bakri in [Bak12] and Zhu in [Zhu16] by different methods, since the exponent of the norm of potential function $V_{0}(x)$ matches the one for eigenfunctions in Donnelly and Fefferman's work.

If $V_{0}(x) \in L^{\infty}$, Bourgain and Kenig [BK05] considered a similar problem for (1.3) motivated by their work on Anderson localization for the Bernoulli model. Bourgain and Kenig established that

$$
\|u\|_{L^{\infty}\left(\mathbb{B}_{r}\right)} \geq c_{1} r^{c_{2}\left(1+\left\|V_{0}\right\|_{L^{\infty}}^{\frac{2}{3}}\right)} \quad \text { as } r \rightarrow 0,
$$

where $c_{1}, c_{2}$ depend only on $n, \hat{C}$. The estimate (1.4) shows that the order of vanishing for solutions is less than $C\left(1+\left\|V_{0}\right\|_{L^{\infty}}^{\frac{2}{3}}\right)$. Kenig in [Ken07] also pointed out that the exponent $\frac{2}{3}$ of $\left\|V_{0}\right\|_{L^{\infty}}$ is sharp for complex valued $V_{0}$ based on Meshkov's example in [Mes92].

Davey in [Dav14] generalized the quantitative unique continuation result to solutions to more general elliptic equations of the form $\Delta u+V_{1} \cdot \nabla u+V_{0} u=\lambda u$, where $\lambda \in \mathbb{C}$, and $V_{0}, V_{1}$ are complex-valued potential functions with pointwise decay at infinity. Davey proved that the order of vanishing for such solutions is less than $C\left(1+\left\|V_{1}\right\|_{L^{\infty}}^{2}+\left\|V_{0}\right\|_{L^{\infty}}^{2 / 3}\right)$. See also the similar work in [Bak13]. Furthermore, The results in [Dav14] were extended to variable-coefficient operators by Lin and Wang in [LW14].

Based on Donnelly and Fefferman's work on the vanishing order of eigenfunctions, Kenig Ken07] asked if the order of vanishing can be reduced to $C\left(1+\left\|V_{0}\right\|_{L^{\infty}}^{1 / 2}\right)$ for real-valued $u$ and $V_{0}$ for the solutions in (1.3). It is related to a quantitative form of Landis' conjecture in the real-valued setting. In the late 1960s, E.M. Landis conjectured that the bounded solution $u$ to $\Delta u-V_{0} u=0$ in $\mathbb{R}^{n}$ is trivial if $|u(x)| \lesssim \exp \left(-c|x|^{1+}\right)$, where $V_{0}$ is a bounded function. By assuming that the bounded real valued $V_{0}(x)$ to be nonnegative, Landis' conjecture was answered in [KSW15] in $\mathbb{R}^{2}$.

It is known that the strong unique continuation property holds for second order elliptic equation (1.2) with singular lower terms satisfying the integrability condition, i.e.

$$
V_{1} \in L^{t} \text { with } t>n \text { and } V_{0} \in L^{\frac{n}{2}} \text {. }
$$

See e.g. [H85], [JK85], [S90], [Wol92], KT01] for the literature about strong unique continuation property, to just mention a few. Recently, interest has been shifted to know how the singular lower order terms control the order of vanishing of solutions. Kenig and Wang in [KW15] studied the quantitative uniqueness of solutions to second order elliptic equations with a drift term $\Delta u+V_{1} \cdot \nabla u=0$ in $\mathbb{R}^{2}$ using complex analytic tools. They established the vanishing order estimates for solutions in the case that real-valued $V_{1} \in L^{s}\left(\mathbb{R}^{2}\right)$ for some $s \in[2, \infty)$. In [KT16], Klein and Tsang studied quantitative unique continuation for solutions to $\Delta u+V_{0} u=0$ motivated by spectral projection of Schrödinger operators, where $V_{0} \in L^{s}$ for some $s \geq n$. Their tools are Carleman estimates as that in [BK05] and Sobolev imbedding arguments. It seems that their method can not be adapted to study elliptic equations with singular gradient potentials. Very recently, by a new quantitative $L^{p} \rightarrow L^{q}$ Carleman estimates for a range of $p$ and $q$ value, Davey and the author in [DZ17] were able to deal with (1.2) with both singular gradient 
potential $V_{1}$ and singular potential $V_{0}$ for $n \geq 3$. Our results not only work for a larger range of singular potentials and gradient potentials, but also improve the previous results on vanishing order of solutions. For $n=2$, Davey and the author in [DaZ17] further explored the $L^{p} \rightarrow L^{q}$ Carleman estimates developed in [DZ17]. They were able to characterize vanishing order for all admissible singular potentials and gradient potentials, which provides a complete description of quantitative uniqueness for second order elliptic equations in $n=2$.

Higher order elliptic equations are important models in the study of partial differential equations. We assume throughout the paper that $m \geq 2$. A nature question is to study the quantitative uniqueness of higher order elliptic equations. However, it is relatively less explored in the literature. The strong unique continuation property has been well investigated for higher order elliptic equations. See e.g. [CG99], [L07], [CK10], to just mention a few. In particular, this property has been shown for singular potential $V_{0}$ and singular coefficient functions $V_{\alpha}$ in [L07]. The $\alpha$ value up to $\left[\frac{3 m}{2}\right]$ for unique continuation was given by Protter [P60]. The vanishing order for higher order elliptic equations was considered in [Zhu16]. For the model

$$
\triangle^{m} u+V_{0} u=0 \quad \text { in } \mathbb{B}_{10},
$$

it was shown in [Zhu16] that the vanishing order of $u$ is less than $C\left\|V_{0}\right\|_{L^{\infty}}$ for $n \geq 4 m$ by a variant of frequency function. Lin, Nagayasu and Wang studied a different quantitative uniqueness result for higher order elliptic equations in [LNW11], where the vanishing order of solutions was not explicitly provided in term of the potential function $V_{0}$ and the coefficient functions $V_{\alpha}$.

A priori, we assume that $u \in W_{l o c}^{m, 2}\left(\mathbb{B}_{10}\right)$ is a weak solution to (1.1). By regularity theory, it follows that $u \in W_{l o c}^{2 m, 2} \cap L_{l o c}^{\infty}$. In the paper, the notation $\mathbb{B}_{r}\left(x_{0}\right) \subset \mathbb{R}^{n}$ is denoted as the ball of radius $r$ centered at $x_{0}$. When the center is clear in the context, we simply write $\mathbb{B}_{r}$. To fully discuss the vanishing order for higher order elliptic equations, our result is stated as three cases in term of the relation of $n$ and $m$.

Theorem 1. Let $u$ be a solution to (1.1) in $\mathbb{B}_{10}$.

I): In the case of $n>4 m-2$, assume that $s \in\left(\frac{2 n}{3 m}, \infty\right]$. Then the vanishing order of $u$ in $\mathbb{B}_{1}$ is less than $C\left(1+\sum_{|\alpha|=1}^{\alpha_{0}}\left\|V_{\alpha}\right\|_{L^{\infty}}^{\mu}+\left\|V_{0}\right\|_{L^{s}}^{\nu}\right)$. That is, for any $x_{0} \in \mathbb{B}_{1}$ and every $r$ sufficiently small,

where

$$
\|u\|_{L^{\infty}\left(\mathbb{B}_{r}\left(x_{0}\right)\right)} \geq \operatorname{cr}^{C\left(1+\sum_{|\alpha|=1}^{\alpha_{0}}\left\|V_{\alpha}\right\|_{L^{\infty}}^{\mu}+\left\|V_{0}\right\|_{L^{s}}^{\nu}\right)}
$$

$$
\mu=\frac{2}{3 m-2|\alpha|} \quad \text { and } \quad \nu=\frac{2 s}{3 m s-2 n} .
$$

and $c=c(n, m, s, \hat{C}), C=C(n, m, s, \hat{C})$.

II): In the case of $n=4 m-2$, assume that $s \in\left(\frac{4(2 m-1)}{3 m}, \infty\right]$. Then for any sufficiently small constant $\varepsilon>0$, the vanishing order of $u$ in $\mathbb{B}_{1}$ is less than $C\left(1+\sum_{|\alpha|=1}^{\alpha_{0}}\left\|V_{\alpha}\right\|_{L^{\infty}}^{\mu}+\left\|V_{0}\right\|_{L^{s}}^{\tilde{v}^{s}}\right)$. That is, for any $x_{0} \in \mathbb{B}_{1}$ and every $r$ sufficiently small,

$$
\|u\|_{L^{\infty}\left(\mathbb{B}_{r}\left(x_{0}\right)\right)} \geq c r^{C\left(1+\sum_{|\alpha|=1}^{\alpha_{0}}\left\|V_{\alpha}\right\|_{L^{\infty}}^{\mu}+\left\|V_{0}\right\|_{L^{s}}^{\tilde{\nu}}\right)}
$$

where

$$
\mu=\frac{2}{3 m-2|\alpha|} \quad \text { and } \quad \tilde{\nu}=\frac{2 s}{3 m s-4(2 m-1)-2(2 m-1)(s-2) \epsilon},
$$

and $c=c(n, m, s, \varepsilon, \hat{C}), C=C(n, m, s, \varepsilon, \hat{C})$.

III): In the case $2 \leq n<4 m-2$, assume that $s \in\left(\frac{4(2 m-1)}{3 m}, \infty\right]$. Then the vanishing order of $u$ in $\mathbb{B}_{1}$ is less than $C\left(1+\sum_{|\alpha|=1}^{\alpha_{0}}\left\|V_{\alpha}\right\|_{L^{\infty}}^{\mu}+\left\|V_{0}\right\|_{L^{s}}^{\bar{\nu}}\right)$. That is, for any $x_{0} \in \mathbb{B}_{1}$ and every $r$ sufficiently small,

$$
\|u\|_{L^{\infty}\left(\mathbb{B}_{r}\left(x_{0}\right)\right)} \geq c r^{C\left(1+\sum_{|\alpha|=1}^{\alpha_{0}}\left\|V_{\alpha}\right\|_{L^{\infty}}^{\mu}+\left\|V_{0}\right\|_{L^{s}}^{\bar{\nu}}\right)}
$$


where

$$
\mu=\frac{2}{3 m-2|\alpha|} \quad \text { and } \quad \bar{\nu}=\frac{2 s}{3 m s-4(2 m-1)},
$$

and $c=c(n, m, s, \hat{C}), C=C(n, m, s, \hat{C})$.

Before we proceed, let us give some comments on Theorem 1.

Remark 1. 1. The vanishing order of solution is heavily relied on the Carleman estimates in Theorem 3, which is split into three cases. To obtain the Carleman estimates for singular weights in suitable Lebesgue spaces, Sobolev inequalities are used in the arguments. The application of Sobolev embedding implies those cases by the relation of $n$ and $m$.

2. In [Zhu16], the author developed a variant of frequency function to obtain the vanishing order less than $C\left\|V_{0}\right\|_{L^{\infty}}$ for $n \geq 4 m$ in (1.5). In addition to the situation $n \geq 4 m$, Theorem 1 provides the description of vanishing order for all cases. Observe that the vanishing order of solution is less than $C\left\|V_{0}\right\|_{L^{\infty}}^{\frac{2}{3 m}}$ if $V_{\alpha}=0$ and $s=\infty$. Theorem 1 not only improves the vanishing order in the case of $s=\infty$ in [Zhu16], but also enables us to deal with singular potential $V_{0}$ and non-trivial coefficient function $V_{\alpha}$.

3. Because of the rich results for second order elliptic equation in the case of $m=1$ in (1.1), we assume $m \geq 2$ in the paper. However, the statement in Case I and II still applies to the case $m=1$. Observe that those conclusions match the sharp results by [BK05] in the case of $s=\infty$ and $m=1$.

Based on the result of vanishing order, one can show the quantitative unique continuation at infinity. The quantitative unique continuation at infinity is characterized by a lower bound for $\mathcal{M}(R)$, where

$$
\mathcal{M}(R):=\inf _{\left|x_{0}\right|=R_{\mathbb{B}_{1}\left(x_{0}\right)}} \sup _{|u(x)| .}
$$

For the equation (1.3) in $\mathbb{R}^{n}$, it is shown in [BK05] that

$$
\mathcal{M}(R) \geq c \exp \left[-C R^{\frac{4}{3}} \log R\right]
$$

from a scaling argument using the estimates (1.4). We are able to show the following characterization of solution at infinity for higher order elliptic equations.

Theorem 2. Assume that $\left\|V_{\alpha}\right\|_{L^{\infty}\left(\mathbb{R}^{n}\right)} \leq A_{\alpha}$ and $\left\|V_{0}\right\|_{L^{s}\left(\mathbb{R}^{n}\right)} \leq A_{0}$. Let $u$ be a solution to (1.1) in $\mathbb{R}^{n}$. Assume that $\|u\|_{L^{\infty}\left(\mathbb{R}^{n}\right)} \leq C_{0}$ and $|u(0)| \geq 1$.

I): In the case of $n>4 m-2$, assume that $s \in\left(\frac{2 n}{3 m}, \infty\right]$. Then for $R>>1$,

$$
\mathcal{M}(R) \geq c \exp \left[-C R^{\Theta} \log R\right]
$$

where $\Theta=\left\{\begin{array}{ll}\frac{2\left(2 m-\alpha_{0}\right)}{3 m-2 \alpha_{0}} & \alpha_{0} \geq \frac{n}{s} \\ \frac{2(2 m s-n)}{3 m s-2 n} & \alpha_{0}<\frac{n}{s}\end{array}, c=c\left(n, m, s, C_{0}\right)\right.$, and $C=C\left(n, m, s, C_{0}, A_{0}, \cdots, A_{\alpha_{0}}\right)$.

II): In the case of $n=4 m-2$, assume that $s \in\left(\frac{4(2 m-1)}{3 m}, \infty\right]$, Then for any sufficiently small constant $\varepsilon>0$ and $R>>1$,

$$
\begin{aligned}
& \text { where } \tilde{\Theta}=\left\{\begin{array}{ll}
\frac{2\left(2 m-\alpha_{0}\right)}{3 m-2 \alpha_{0}} & \alpha_{0} \geq \frac{8 m(2 m-1)-3 m n+4 m(2 m-1)(s-2) \epsilon}{m s+4(2 m-1)-2 n+2(2 m-1)(s-2) \epsilon} \\
\frac{2(2 m s-n)}{3 m s-4(2 m-1)-2(2 m-1)(s-2) \epsilon} & \alpha_{0}<\frac{8 m(2 m-1)-3 m n+4 m(2 m-1)(s-2) \epsilon}{m s+4(2 m-1)-2 n+2(2 m-1)(s-2) \epsilon}
\end{array},\right. \\
& c=c\left(n, s, m, \varepsilon, C_{0}\right), \text { and } C=C\left(n, s, m, \varepsilon, C_{0}, A_{0}, \cdots, A_{\alpha_{0}}\right) .
\end{aligned}
$$


III): In the case of $2 \leq n<4 m-2$, assume that $s \in\left(\frac{4(2 m-1)}{3 m}, \infty\right]$. Then for $R>>1$,

$$
\begin{gathered}
\mathcal{M}(R) \geq c \exp \left[-C R^{\bar{\Theta}} \log R\right], \\
\text { where } \bar{\Theta}= \begin{cases}\frac{2\left(2 m-\alpha_{0}\right)}{3 m-2 \alpha_{0}} & \alpha_{0} \geq \frac{8 m(2 m-1)-3 m n}{m s+4(2 m-1)-2 n} \\
\frac{2(2 m s-n)}{3 m s-4(2 m-1)} & \alpha_{0}<\frac{8 m(2 m-1)-3 m n}{m s+4(2 m-1)-2 n}\end{cases} \\
c=c\left(n, s, m, C_{0}\right), \text { and } C=C\left(n, s, m, C_{0}, A_{0}, \cdots, A_{\alpha_{0}}\right) .
\end{gathered}
$$

Remark 2. 1. In particular, the vanishing at infinity as (1.7) for the higher order elliptic equation (1.5) with $V_{0} \in L^{\infty}$ was shown in [HWZ16]. If $s=\infty$ and $V_{\alpha}=0$, our Theorem will implies (1.7) as well. Obviously, the results in [HWZ16] is just a special case of Theorem Q. The work enables us to deal with the singular potential $V_{0}$ as well as the presence of coefficient function $V_{\alpha}$ for the results of vanishing at infinity.

2. The case $I$ and II in Theorem 2 also work for the case $m=1$. If $V_{\alpha}=0, m=1$ and $s=\infty$, the conclusions match the sharp result (1.7) for the seconder order elliptic equation in [BK05]. Clearly, we have obtained the results for much more general cases.

Generally speaking, the frequency function and Carleman estimates are two major ways to obtain qualitative and quantitative unique continuation results for solutions of partial differential equations. The frequency function describes the local growth rate of $u$ and is considered as a local measure of its "degree" for a polynomial like function in $\mathbb{B}_{r}$. See e.g. [GL86], GL87], [Ku98], Zhu16] for the application of frequency function, to just mention a few. Carleman estimates are weighted integral inequalities. To obtain the quantitative uniqueness results for solutions, one usually uses the Carleman estimates with a special choice of weight functions to obtain some type of Hadamard's three-ball theorem, then employ "propagation of smallness" argument to obtain maximal order of vanishing. In this paper, we establish a new quantitative $L^{2} \rightarrow L^{p}$ Carleman inequalities with a range of $p$ value for higher order elliptic operators. We first derive a quantitative $L^{2} \rightarrow L^{2}$ Carleman inequalities involving terms for every order derivative for second order elliptic operators. Then, using an iterative procedure, we obtain a quantitative $L^{2} \rightarrow L^{2}$ Carleman inequalities for higher order elliptic operators. The $L^{2} \rightarrow L^{p}$ Carleman estimates are attained from Sobolev embedding and an interpolation argument, which adapts the idea in [DZ17].

Let us comment on the organization of the article. Section 2 is devoted to obtaining Carleman estimates for the higher order elliptic operators with singular potential functions $V_{0}$ and coefficient functions $V_{\alpha}$. In Section 3, the main tool $L^{2} \rightarrow L^{p}$ Carleman estimates are established. We also derive some type of quantitative Caccioppoli inequality and $L^{\infty}$ type estimates for higher order elliptic equations. In section 4, we deduce three-ball inequalities from the Carleman estimates and obtain the vanishing order estimates from the propagation of smallness argument. The proof of Theorem 2 is presented in Section [5. The letters $c, C, C_{0}$ and $C_{1}$ denote generic positive constants that do not depends on $u$, and may vary from line to line. In the paper, the norm $\left\|V_{0}\right\|_{L^{s}}$ and $\left\|V_{\alpha}\right\|_{L^{\infty}}$ are assumed to be sufficiently large. Otherwise, we may assume that $\left\|V_{0}\right\|_{L^{s}} \leq M_{0}$ and $\left\|V_{\alpha}\right\|_{L^{\infty}} \leq M_{\alpha}$ for some sufficiently large $M_{0}$ and $M_{\alpha}$. Then we may replace the $\left\|V_{0}\right\|_{L^{s}}$ by $M_{0}$ and the $\left\|V_{\alpha}\right\|_{L^{\infty}}$ by $M_{\alpha}$ in Theorem 1,

\section{Carleman estimates}

In this section, we state the crucial tools, the quantitative Carleman estimates. Set

$$
\phi(r)=\log r+\log (\log r)^{2} .
$$


Let $r=\left|x-x_{0}\right|$. We use the notation $\|u\|_{L^{p}\left(r^{-n} d x\right)}$ to denote the $L^{p}$ norm with weight $r^{-n}$, i.e.

$$
\|u\|_{L^{p}\left(r^{-n} d x\right)}=\left(\int|u|^{p} r^{-n} d x\right)^{\frac{1}{p}} .
$$

Our quantitative Carleman estimate for the higher order elliptic operators is stated as follows. Three cases are discussed in term of the relation of $n$ and $m$.

Theorem 3. (I): In the case of $n>4 m-2$ and $2 \leq p \leq \frac{2 n}{n-4 m+2}$, there exist a constant $C$ and a sufficiently small $R_{0}$ such that for any $u \in C_{0}^{\infty}\left(\mathbb{B}_{R_{0}}\left(x_{0}\right) \backslash\left\{x_{0}\right\}\right)$ and $\tau>1$, one has

$$
\begin{aligned}
& \tau^{\beta_{0}}\left\|e^{-\tau \phi(r)}(\log r)^{-m} u\right\|_{L^{p}\left(r^{-n} d x\right)}+\sum_{|\alpha|=1}^{2 m-1} \tau^{\beta_{\alpha}}\left\|e^{-\tau \phi(r)}(\log r)^{-m} r^{|\alpha|} D^{\alpha} u\right\|_{L^{2}\left(r^{-n} d x\right)} \\
& \leq C\left\|e^{-\tau \phi(r)} r^{2 m} \triangle^{m} u\right\|_{L^{2}\left(r^{-n} d x\right)},
\end{aligned}
$$

where $\beta_{0}=\frac{3 m p-n(p-2)}{2 p}$ and $\beta_{\alpha}=\frac{3 m-2|\alpha|}{2}$.

(II): In the case of $n=4 m-2$ and $2 \leq p<\infty$, there exist a constant $C$ and a sufficiently small $R_{0}$ such that for any $u \in C_{0}^{\infty}\left(\mathbb{B}_{R_{0}}\left(x_{0}\right) \backslash\left\{x_{0}\right\}\right)$ and $\tau>1$, one has

$$
\begin{aligned}
& \tau^{\tilde{\beta}_{0}}\left\|e^{-\tau \phi(r)}(\log r)^{-m} u\right\|_{L^{p}}+\sum_{|\alpha|=0}^{2 m-1} \tau^{\beta_{\alpha}}\left\|e^{-\tau \phi(r)}(\log r)^{-m} r^{|\alpha|} D^{\alpha} u\right\|_{L^{2}\left(r^{-n} d x\right)} \\
& \leq C\left\|e^{-\tau \phi(r)} r^{2 m} \triangle^{m} u\right\|_{L^{2}\left(r^{-n} d x\right)},
\end{aligned}
$$

where $\tilde{\beta}_{0}=\frac{4 m-2}{p}(1-\varepsilon)-\frac{m-2}{2}, \beta_{\alpha}=\frac{3 m-2|\alpha|}{2}$, and $0<\epsilon<1$ is sufficiently small.

(III): In the case of $2 \leq n<4 m-2$ and $2 \leq p \leq \infty$ there exist a constant $C$ and a sufficiently small $R_{0}$ such that for any $u \in C_{0}^{\infty}\left(\mathbb{B}_{R_{0}}\left(x_{0}\right) \backslash\left\{x_{0}\right\}\right)$ and $\tau>1$, one has

$$
\begin{aligned}
& \tau^{\bar{\beta}_{0}}\left\|e^{-\tau \phi(r)}(\log r)^{-m} u\right\|_{L^{p}}+\sum_{|\alpha|=0}^{2 m-1} \tau^{\beta_{\alpha}}\left\|e^{-\tau \phi(r)}(\log r)^{-m} r^{|\alpha|} D^{\alpha} u\right\|_{L^{2}\left(r^{-n} d x\right)} \\
& \leq C\left\|e^{-\tau \phi(r)} r^{2 m} \triangle^{m} u\right\|_{L^{2}\left(r^{-n} d x\right)},
\end{aligned}
$$

where $\bar{\beta}_{0}=\frac{4 m-2}{p}-\frac{m-2}{2}, \beta_{\alpha}=\frac{3 m-2|\alpha|}{2}$.

We provide the proof of Theorem 3 in the next section. We are going to use Theorem 3 to establish the following Carleman estimates for higher order elliptic equations of the form (1.1). For an appropriate choice of $p$ and sufficiently large $\tau$, from Theorem 3, we replace the higher order elliptic operator with a higher order elliptic operator with potential functions $V_{0}$ and coefficient functions $V_{\alpha}$ using Hölder's inequality and the triangle inequality.

Theorem 4. I): In the case of $n>4 m-2$, assume that $s \in\left(\frac{2 n}{3 m}, \infty\right]$, there exist constants $C_{0}$, $C_{1}$, and sufficiently small $R_{0}<1$ such that for any $u \in C_{0}^{\infty}\left(\mathbb{B}_{R_{0}}\left(x_{0}\right) \backslash\left\{x_{0}\right\}\right)$ and large positive constant

$$
\tau>C_{0}\left(1+\sum_{|\alpha|=1}^{\alpha_{0}}\left\|V_{\alpha}\right\|_{L^{\infty}}^{\mu}+\left\|V_{0}\right\|_{L^{s}}^{\nu}\right),
$$

one has

$$
\begin{aligned}
& \tau^{\beta_{0}}\left\|e^{-\tau \phi(r)}(\log r)^{-m} u\right\|_{L^{p}\left(r^{-n} d x\right)}+\sum_{|\alpha|=1}^{2 m-1} \tau^{\beta_{\alpha}}\left\|e^{-\tau \phi(r)}(\log r)^{-m} r^{|\alpha|} D^{\alpha} u\right\|_{L^{2}\left(r^{-n} d x\right)} \\
& \leq C_{1}\left\|e^{-\tau \phi(r)} r^{2 m}\left(\triangle^{m} u+\sum_{|\alpha|=1}^{\alpha_{0}} V_{\alpha} D^{\alpha} u+V_{0} u\right)\right\|_{L^{2}\left(r^{-n} d x\right)},
\end{aligned}
$$


where

$$
\mu=\frac{2}{3 m-2|\alpha|} \quad \text { and } \quad \nu=\frac{2 s}{3 m s-2 n}
$$

and $\beta_{0}, \beta_{\alpha}$ and $p$ as defined in Theorem 3 .

II:) In the case $n=4 m-2$, assume that $s \in\left(\frac{4(2 m-1)}{3 m}, \infty\right]$, there exist constants $C_{0}, C_{1}$, and sufficiently small $R_{0}<1$ such that for any $u \in C_{0}^{\infty}\left(\mathbb{B}_{R_{0}}\left(x_{0}\right) \backslash\left\{x_{0}\right\}\right)$ and large positive constant

$$
\tau>C_{0}\left(1+\sum_{|\alpha|=1}^{\alpha_{0}}\left\|V_{\alpha}\right\|_{L^{\infty}}^{\mu}+\left\|V_{0}\right\|_{L^{s}}^{\tilde{\nu}^{s}}\right)
$$

one has

$$
\begin{aligned}
& \tau^{\tilde{\beta}_{0}}\left\|e^{-\tau \phi(r)}(\log r)^{-m} u\right\|_{L^{p}}+\sum_{|\alpha|=1}^{2 m-1} \tau^{\beta_{\alpha}}\left\|e^{-\tau \phi(r)}(\log r)^{-m} r^{|\alpha|} D^{\alpha} u\right\|_{L^{2}\left(r^{-n} d x\right)} \\
& \leq C_{1}\left\|e^{-\tau \phi(r)} r^{2 m}\left(\triangle^{m} u+\sum_{|\alpha|=1}^{\alpha_{0}} V_{\alpha} D^{\alpha} u+V_{0} u\right)\right\|_{L^{2}\left(r^{-n} d x\right)},
\end{aligned}
$$

where

$$
\mu=\frac{2}{3 m-2|\alpha|} \quad \text { and } \quad \tilde{\nu}=\frac{2 s}{3 m s-4(2 m-1)-2(2 m-1)(s-2) \epsilon}
$$

and $\tilde{\beta}_{0}, \beta_{\alpha}$ and $p$ as defined in Theorem 3 .

III:) In the case $2 \leq n<4 m-2$, assume that $s \in\left(\frac{4(2 m-1)}{3 m}, \infty\right]$, there exist constants $C_{0}$, $C_{1}$, and sufficiently small $R_{0}<1$ such that for any $u \in C_{0}^{\infty}\left(\mathbb{B}_{R_{0}}\left(x_{0}\right) \backslash\left\{x_{0}\right\}\right)$ and large positive constant

$$
\tau>C_{0}\left(1+\sum_{|\alpha|=1}^{\alpha_{0}}\left\|V_{\alpha}\right\|_{L^{\infty}}^{\mu}+\left\|V_{0}\right\|_{L^{s}}^{\bar{\nu}}\right)
$$

one has

$$
\begin{aligned}
& \tau^{\bar{\beta}_{0}}\left\|e^{-\tau \phi(r)}(\log r)^{-m} u\right\|_{L^{p}}+\sum_{|\alpha|=1}^{2 m-1} \tau^{\beta_{\alpha}}\left\|e^{-\tau \phi(r)}(\log r)^{-m} r^{|\alpha|} D^{\alpha} u\right\|_{L^{2}\left(r^{-n} d x\right)} \\
& \leq C_{1}\left\|e^{-\tau \phi(r)} r^{2 m}\left(\triangle^{m} u+\sum_{|\alpha|=1}^{\alpha_{0}} V_{\alpha} D^{\alpha} u+V_{0} u\right)\right\|_{L^{2}\left(r^{-n} d x\right)},
\end{aligned}
$$

where

$$
\mu=\frac{2}{3 m-2|\alpha|} \quad \text { and } \quad \bar{\nu}=\frac{2 s}{3 m s-4(2 m-1)},
$$

and $\bar{\beta}_{0}, \beta_{\alpha}$ and $p$ as defined in Theorem 3 . 
Proof. Case I): We first consider the case $n>4 m-2$. With the aid of Theorem 3 and the triangle inequality, we see that

$$
\begin{aligned}
& \tau^{\beta_{0}}\left\|e^{-\tau \phi(r)}(\log r)^{-m} u\right\|_{L^{p}\left(r^{-n} d x\right)}+\sum_{|\alpha|=1}^{2 m-1} \tau^{\beta_{\alpha}}\left\|e^{-\tau \phi(r)}(\log r)^{-m} r^{|\alpha|} D^{\alpha} u\right\|_{L^{2}\left(r^{-n} d x\right)} \\
& \leq C\left\|e^{-\tau \phi(r)} r^{2 m} \triangle^{m} u\right\|_{L^{2}\left(r^{-n} d x\right)} \\
& \leq C\left\|e^{-\tau \phi(r)} r^{2 m}\left(\triangle^{m} u+\sum_{|\alpha|=1}^{\alpha_{0}} V_{\alpha} D^{\alpha} u+V_{0} u\right)\right\|_{L^{2}\left(r^{-n} d x\right)} \\
& +\sum_{|\alpha|=1}^{\alpha_{0}} C\left\|e^{-\tau \phi(r)} r^{2 m} V_{\alpha} D^{\alpha} u\right\|_{L^{2}\left(r^{-n} d x\right)} \\
& +C\left\|e^{-\tau \phi(r)} r^{2 m} V_{0} u\right\|_{L^{2}\left(r^{-n} d x\right)} .
\end{aligned}
$$

Now we estimate the last two terms in the right hand side of (2.7). Set $p=\frac{2 s}{s-2}$. Note that $m \geq 2$. By the assumption that $n>4 m-2$, we obtain $s>\frac{2 n}{3 m}>2$. Thus, $p$ is a positive constant. Since $\frac{n}{2 m-1} \leq \frac{2 n}{3 m}<s \leq \infty$, then $2 \leq p<\frac{2 n}{n-3 m} \leq \frac{2 n}{n-4 m+2}$, which in the range of $p$ in Theorem 3. Following from Hölder's inequality, we obtain that

$$
\begin{aligned}
& \left\|e^{-\tau \phi(r)} r^{2 m} V_{0} u\right\|_{L^{2}\left(r^{-n} d x\right)} \\
& \leq\left\|V_{0}\right\|_{L^{s}\left(B_{R_{0}}\right)}\left\|e^{-\tau \phi(r)} r^{2 m+\frac{n}{p}-\frac{n}{2}} u\right\|_{L^{p}\left(r^{-n} d x\right)} \\
& \leq\left\|V_{0}\right\|_{L^{s}\left(B_{R_{0}}\right)}\left\|e^{-\tau \phi(r)}(\log r)^{-m} u\right\|_{L^{p}\left(r^{-n} d x\right)}\left\|(\log r)^{m} r^{2 m+\frac{n}{p}-\frac{n}{2}}\right\|_{L^{\infty}\left(B_{R_{0}}\right)} \\
& \leq C\left\|V_{0}\right\|_{L^{s}\left(B_{R_{0}}\right)}\left\|e^{-\tau \phi(r)}(\log r)^{-m} u\right\|_{L^{p}\left(r^{-n} d x\right)},
\end{aligned}
$$

where we have used the fact that $2 m+\frac{n}{p}-\frac{n}{2}>0$ and $R_{0}$ is small. Furthermore, using Hölder's inequality,

$$
\begin{aligned}
& \sum_{|\alpha|=1}^{\alpha_{0}}\left\|e^{-\tau \phi(r)} r^{2 m} V_{\alpha} D^{\alpha} u\right\|_{L^{2}\left(r^{-n} d x\right)} \\
& \leq \sum_{|\alpha|=1}^{\alpha_{0}}\left\|V_{\alpha}\right\|_{L^{\infty}}\left\|r^{2 m-|\alpha|}(\log r)^{m}\right\|_{L^{\infty}}\left\|e^{-\tau \phi(r)}(\log r)^{-m} r^{|\alpha|} D^{\alpha} u\right\|_{L^{2}\left(r^{-n} d x\right)} \\
& \leq \sum_{|\alpha|=1}^{\alpha_{0}}\left\|V_{\alpha}\right\|_{L^{\infty}}\left\|e^{-\tau \phi(r)}(\log r)^{-m} r^{|\alpha|} D^{\alpha} u\right\|_{L^{2}\left(r^{-n} d x\right)} .
\end{aligned}
$$

In order to absorb the last two terms in the right hand side of (2.7) into the the left hand side, from (2.9) and (2.8), we choose

$$
\tau^{\beta_{\alpha}} \geq c\left\|V_{\alpha}\right\|_{L^{\infty}} \quad \text { and } \quad \tau^{\beta_{0}} \geq c\left\|V_{0}\right\|_{L^{s}}
$$

From the assumption of $\alpha_{0}$, we know $\beta_{\alpha}=\frac{3 m-2|\alpha|}{2}>0$. We can check that $\beta_{0}=\frac{3 m p-n(p-2)}{2 p}=$ $\frac{3 m s-2 n}{2 s}$. Because $s>\frac{2 n}{3 m}$, we see that $\beta_{0}>0$.

Therefore, to reach (2.10), we need to choose

$$
\tau>C\left(1+\sum_{|\alpha|=1}^{\alpha_{0}}\left\|V_{\alpha}\right\|_{L^{\infty}}^{\mu}+\left\|V_{0}\right\|_{L^{p}}^{\nu}\right)
$$

where

$$
\mu=\frac{2}{3 m-2|\alpha|} \quad \text { and } \quad \nu=\frac{2 s}{3 m s-2 n} .
$$


Case II): Now we turn to the case of $n=4 m-2$. Carrying out the similar arguments as (2.7), we obtain

$$
\begin{aligned}
& \tau^{\tilde{\beta}_{0}}\left\|e^{-\tau \phi(r)}(\log r)^{-m} u\right\|_{L^{p}}+\sum_{|\alpha|=0}^{2 m-1} \tau^{\beta_{\alpha}}\left\|e^{-\tau \phi(r)}(\log r)^{-m} r^{|\alpha|} D^{\alpha} u\right\|_{L^{2}\left(r^{-n} d x\right)} \\
& \leq C\left\|e^{-\tau \phi(r)} r^{2 m}\left(\triangle^{m} u+\sum_{|\alpha|=1}^{\alpha_{0}} V_{\alpha} D^{\alpha} u+V_{0} u\right)\right\|_{L^{2}\left(r^{-n} d x\right)} \\
& \left.+\sum_{|\alpha|=1}^{\alpha_{0}} C\left\|e^{-\tau \phi(r)} r^{2 m} V_{\alpha} D^{\alpha} u\right\|_{L^{2}\left(r^{-n}\right.} d x\right) \\
& +C\left\|e^{-\tau \phi(r)} r^{2 m} V_{0} u\right\|_{L^{2}\left(r^{-n} d x\right)} .
\end{aligned}
$$

Set $p=\frac{2 s}{s-2}$ again. Since $s>\frac{4(2 m-1)}{3 m}$, we check that $s>2$. It follows from Hölder's inequality that

$$
\begin{aligned}
& \left\|e^{-\tau \phi(r)} r^{2 m} V_{0} u\right\|_{L^{2}\left(r^{-n} d x\right)} \\
& \leq\left\|V_{0}\right\|_{L^{s}\left(B_{R_{0}}\right)}\left\|e^{-\tau \phi(r)} r^{2 m-\frac{n}{2}} u\right\|_{L^{p}} \\
& \leq\left\|V_{0}\right\|_{L^{s}\left(B_{R_{0}}\right)}\left\|e^{-\tau \phi(r)}(\log r)^{-m} u\right\|_{L^{p}}\left\|(\log r)^{m} r^{2 m-\frac{n}{2}}\right\|_{L^{\infty}\left(B_{R_{0}}\right)} \\
& \leq C\left\|V_{0}\right\|_{L^{s}\left(B_{R_{0}}\right.}\left\|e^{-\tau \phi(r)}(\log r)^{-m} u\right\|_{L^{p}},
\end{aligned}
$$

where we have considered that $2 m-\frac{n}{2}>0$. For the terms involving higher order derivatives, we carry out the the same argument as (2.9). We can also absorb the last two terms in the right hand side of (2.11) into the left hand side. Together with (2.12) and (2.9), we choose

$$
\tau^{\beta_{\alpha}} \geq c\left\|V_{\alpha}\right\|_{L^{\infty}} \quad \text { and } \quad \tau^{\tilde{\beta}_{0}} \geq c\left\|V_{0}\right\|_{L^{s}}
$$

Since $s>\frac{4(2 m-1)}{3 m}$, we can check that

$$
\tilde{\beta}_{0}=\frac{2(2 m-1)}{p}(1-\epsilon)-\frac{m-2}{2}=\frac{3 m s-4(2 m-1)-2(2 m-1)(s-2) \varepsilon}{2 s}>0
$$

by choosing $\epsilon$ sufficiently small.

To satisfy (2.13), we choose

$$
\tau>C\left(1+\sum_{|\alpha|=1}^{\alpha_{0}}\left\|V_{\alpha}\right\|_{L^{\infty}}^{\mu}+\left\|V_{0}\right\|_{L^{p}}^{\tilde{\nu}^{p}}\right)
$$

with

$$
\mu=\frac{2}{3 m-2|\alpha|} \quad \text { and } \quad \tilde{\nu}=\frac{2 s}{3 m s-4(2 m-1)-2(2 m-1)(s-2) \epsilon},
$$

where $0<\epsilon<1$ is sufficiently small. 
Case III): At last, we deal with the case $2 \leq n<4 m-2$. Similar to the argument in Case II, by triangle inequality and Hölder's inequality, it follows from Theorem 3 that

$$
\begin{aligned}
& \tau^{\bar{\beta}_{0}}\left\|e^{-\tau \phi(r)}(\log r)^{-m} u\right\|_{L^{p}}+\sum_{|\alpha|=0}^{2 m-1} \tau^{\beta_{\alpha}}\left\|e^{-\tau \phi(r)}(\log r)^{-m} r^{|\alpha|} D^{\alpha} u\right\|_{L^{2}\left(r^{-n} d x\right)} \\
& \leq C\left\|e^{-\tau \phi(r)} r^{2 m}\left(\triangle^{m} u+\sum_{|\alpha|=1}^{\alpha_{0}} V_{\alpha} D^{\alpha} u+V_{0} u\right)\right\|_{L^{2}\left(r^{-n} d x\right)} \\
& +\sum_{|\alpha|=1}^{\alpha_{0}} C\left\|V_{\alpha}\right\|_{L^{\infty}}\left\|e^{-\tau \phi(r)} r^{2 m} D^{\alpha} u\right\|_{L^{2}\left(r^{-n} d x\right)} \\
& \left.+C\left\|e^{-\tau \phi(r)} r^{2 m} V_{0} u\right\|_{L^{2}\left(r^{-n}\right.} d x\right)
\end{aligned}
$$

and

$$
\left\|e^{-\tau \phi(r)} r^{2 m} V_{0} u\right\|_{L^{2}\left(r^{-n} d x\right)} \leq C\left\|V_{0}\right\|_{L^{s}\left(B_{R_{0}}\right)}\left\|e^{-\tau \phi(r)}(\log r)^{-m} u\right\|_{L^{p}}
$$

for $p=\frac{2 s}{s-2}$. We choose

$$
\tau^{\beta_{\alpha}} \geq c\left\|V_{\alpha}\right\|_{L^{\infty}} \quad \text { and } \quad \tau^{\bar{\beta}_{0}} \geq c\left\|V_{0}\right\|_{L^{s}}
$$

since $s \in\left(\frac{4(2 m-1)}{3 m}, \infty\right]$, we can verify that

$$
\bar{\beta}_{0}=\frac{3 m s-4(2 m-1)}{2 s}>0 .
$$

To satisfy (2.16), we select

$$
\tau>C\left(1+\sum_{|\alpha|=1}^{\alpha_{0}}\left\|V_{\alpha}\right\|_{L^{\infty}}^{\mu}+\left\|V_{0}\right\|_{L^{s}}^{\bar{\nu}}\right),
$$

where $\mu=\frac{2}{3 m-2|\alpha|}$ and $\bar{\nu}=\frac{2 s}{3 m s-4(2 m-1)}$. Thus, the estimate (2.6) is achieved.

Together with the discussion in those three cases, we complete the proof of Theorem 3

\section{Proof of Carleman estimates}

In this section, we prove the crucial tool in the whole paper, i.e. the $L^{2}-L^{p}$ Carleman estimate stated in Theorem 3. To prove our Carleman estimate, we first establish a $L^{2}$ type Carleman estimates for higher order elliptic operators.

We introduce polar coordinates in $\mathbb{R}^{n} \backslash\{0\}$ by setting $x=r \omega$, with $r=|x|$ and $\omega=$ $\left(\omega_{1}, \cdots, \omega_{n}\right) \in S^{n-1}$. Further, we use a new coordinate $t=\log r$. Then

$$
\frac{\partial}{\partial x_{j}}=e^{-t}\left(\omega_{j} \partial_{t}+\Omega_{j}\right), \quad 1 \leq j \leq n,
$$

where $\Omega_{j}$ is a vector field in $S^{n-1}$. It is well known that vector fields $\Omega_{j}$ satisfy

$$
\sum_{j=1}^{n} \omega_{j} \Omega_{j}=0 \quad \text { and } \quad \sum_{j=1}^{n} \Omega_{j} \omega_{j}=n-1 .
$$

The adjoint of $\Omega_{j}$ is an operator in $L\left(S^{n-1}\right)$ given by

$$
\Omega_{j}^{*}=(n-1) \omega_{j}-\Omega_{j} .
$$

It is known that

$$
\sum_{j=1}^{n} \Omega_{j}^{*} \Omega_{j}=-\triangle_{\omega}
$$


We denote $\Omega^{\alpha}$ as the product of $\Omega_{1}^{\alpha_{1}} \cdots \Omega_{n}^{\alpha_{n}}$, where $\alpha=\left(\alpha_{1}, \cdots, \alpha_{n}\right) \in \mathbb{N}^{n}$.

We are interested in $\phi(r) \in C_{0}^{\infty}\left(B_{R_{0}}\left(x_{0}\right) \backslash\left\{x_{0}\right\}\right)$ for some small $R_{0}$. Since $r=e^{t}$, then $r \rightarrow 0$ if and only if $t \rightarrow-\infty$. In terms of $t$, we consider the case $-\infty<t<t_{0}<0$, where $\left|t_{0}\right|$ is chosen to be sufficiently large. Since

$$
r^{2} \triangle u=r^{2} \partial_{r}^{2} u+(n-1) r \partial_{r} u+\triangle_{\omega} u .
$$

In the new coordinate system, the Laplace operator takes the form

$$
e^{2 t} \triangle u=\partial_{t}^{2} u+(n-2) \partial_{t} u+\triangle_{\omega} u,
$$

where $\triangle_{\omega}=\sum_{j=1}^{n} \Omega_{j}^{2}$ is the Laplace-Beltrami operator on $S^{n-1}$.

The idea of establishing the following Carleman estimates is motivated by [R97] and [L07]. However, the test function $\phi(r)=\frac{\tau}{2}(\log r)^{2}$ chosen in [R97] and [L07] is not a log linear function. The log linearity of test functions is essential in deriving the vanishing order in section 4 . In the following proposition, we choose a $\log$ linear function $\phi(r)=\log r+\log (\log r)^{2}$. More delicate analysis is devoted to establishing the estimates.

Proposition 1. Given $\sigma_{1} \in \mathbb{Z}$ and $\sigma_{2} \in \mathbb{Z}$. Then there exist positive constant $C$, large positive constant $\tau_{0}$, and sufficiently small $R_{0}<1$ such that for any $u \in C_{0}^{\infty}\left(\mathbb{B}_{R_{0}}\left(x_{0}\right) \backslash\left\{x_{0}\right\}\right)$, one has

$$
\begin{array}{r}
\left.\sum_{|\alpha|=0}^{2 m-1} \tau^{\beta_{\alpha}}\left\|e^{-\tau \phi(r)}(\log r)^{\sigma_{2}-m} r^{\sigma_{1}+|\alpha|}\left|D^{\alpha} u\right|\right\|_{L^{2}\left(r^{-n}\right.} d x\right) \\
\left.\leq C\left\|e^{-\tau \phi(r)}(\log r)^{\sigma_{2}} r^{\sigma_{1}+2 m} \triangle^{m} u\right\|_{L^{2}\left(r^{-n}\right.} d x\right)
\end{array}
$$

for $\tau>\tau_{0}$, where $\beta_{\alpha}=\frac{3 m-2|\alpha|}{2}$.

Proof. Our strategy is to prove a $L^{2}$ type Carleman estimates for second order elliptic operator. Then we can perform an interative process to get the Carleman estimates for higher order elliptic operators. First, we derive the following Carleman estimates involving terms for every order derivative with weights,

$$
\sum_{|\alpha| \leq 2} \tau^{\frac{3-2|\alpha|}{2}}\left\|e^{-\tau \phi(r)}(\log r)^{\sigma_{2}-1} r^{\sigma_{1}+|\alpha|} D^{\alpha} u\right\|_{L^{2}\left(r^{-n} d x\right)} \leq\left\|e^{-\tau \phi(r)}(\log r)^{\sigma_{2}} r^{\sigma_{1}+2} \triangle u\right\|_{L^{2}\left(r^{-n} d x\right)} .
$$

By the polar coordinates, the right hand side of (3.3) can be written as

$$
\begin{aligned}
\iint e^{-2 \tau \phi(r)} r^{2 \sigma_{1}+4-n}(\log r)^{2 \sigma_{2}}|\triangle u|^{2} d x & =\iint e^{-2 \tau\left(t+\log t^{2}\right)} e^{2 \sigma_{1} t+4 t} t^{2 \sigma_{2}}|\triangle u|^{2} d t d \omega \\
& =\iint\left|e^{-\tau\left(t+\log t^{2}\right)} e^{\sigma_{1} t+2 t} t^{\sigma_{2}} \triangle u\right|^{2} d t d \omega .
\end{aligned}
$$

Let

$$
u=e^{\tau\left(t+\log t^{2}\right)} e^{-\sigma_{1} t} t^{-\sigma_{2}} v .
$$

Direct calculations show that

$$
\begin{aligned}
e^{-\tau\left(t+\ln t^{2}\right)} e^{\sigma_{1} t+2 t} t^{\sigma_{2}} \triangle u & =\left[\partial_{t}+\left(\tau+2 \tau t^{-1}\right)\right]^{2} v+(n-2)\left(\partial_{t}+\left(\tau+2 \tau t^{-1}\right)\right) v \\
& +\triangle_{\omega} v+a \partial_{t} v+b v
\end{aligned}
$$

where $a=-2 \sigma_{1}-\frac{2 \sigma_{2}}{t}$ and

$$
b=-(n-2)\left(\sigma_{1}+\frac{\sigma_{2}}{t}\right)+\frac{\sigma_{2}}{t^{2}}+\left(\sigma_{1}-\frac{\sigma_{2}}{t}\right)^{2}-2\left(\tau+\frac{2 \tau}{t}\right)\left(\sigma_{1}+\frac{\sigma_{2}}{t}\right) .
$$

Note that

$$
a^{\prime}(t)=O\left(t^{-2}\right), \quad \text { and } \quad b^{\prime}(t)=O\left(\tau t^{-2}\right) .
$$


Next we define a new operator by

$$
\triangle_{\tau} v=\left[\partial_{t}+\left(\tau+2 \tau t^{-1}\right)\right]^{2} v+((n-2)+a) \partial_{t} v+\left[(n-2)\left(\tau+2 \tau t^{-1}\right)+b\right] v+\triangle_{\omega} v .
$$

To show (3.3), it is equivalent to obtain that

$$
\sum_{j+|\alpha| \leq 2} \tau^{3-2(j+|\alpha|)} \iint t^{-2}\left|\partial_{t}^{j} \Omega^{\alpha} v\right|^{2} d t d \omega \leq C \iint\left|\triangle_{\tau} v\right|^{2} d t d \omega .
$$

Let $\triangle_{\tau}^{-} v$ be the operator obtained from $\triangle_{\tau} v$ by replacing $\partial_{t}$ with $-\partial_{t}$, i.e.

$$
\triangle_{\tau}^{-} v=\left[\partial_{t}-\left(\tau+2 \tau t^{-1}\right)\right]^{2} v-((n-2)+a) \partial_{t} v+\left[(n-2)\left(\tau+2 \tau t^{-1}\right)+b\right] v+\triangle_{\omega} v .
$$

Note that

$$
\begin{aligned}
\triangle_{\tau} v=\partial_{t}^{2} v & +2\left(\tau+2 \tau t^{-1}\right) \partial_{t} v-2 t^{-2} \tau v+\left(\tau+2 \tau t^{-1}\right)^{2} v+((n-2)+a) \partial_{t} v \\
+ & {\left[(n-2)\left(\tau+2 \tau t^{-1}\right)+b\right] v+\triangle_{\omega} v }
\end{aligned}
$$

Similar calculations show that

$$
\begin{aligned}
\triangle_{\tau}^{-} v=\partial_{t}^{2} v & -2\left(\tau+2 \tau t^{-1}\right) \partial_{t} v+2 t^{-2} \tau v+\left(\tau+2 \tau t^{-1}\right)^{2} v-((n-2)+a) \partial_{t} v \\
+ & {\left[(n-2)\left(\tau+2 \tau t^{-1}\right)+b\right] v+\triangle_{\omega} v . }
\end{aligned}
$$

On one hand, we compute the integration of the difference of $\triangle_{\tau} v$ and $\triangle_{\tau}^{-} v$. Define

$$
I:=\iint\left|\triangle_{\tau} v\right|^{2} d t d \omega-\iint\left|\triangle_{\tau}^{-} v\right|^{2} d t d \omega .
$$

It follows from (3.7) and (3.8) that

$$
\begin{aligned}
I= & 4\left\langle\partial_{t}^{2} v+\left(\tau+2 \tau t^{-1}\right)^{2} v+\left[(n-2)\left(\tau+2 \tau t^{-1}\right)+b\right] v+\triangle_{\omega} v, \quad 2\left(\tau+2 \tau t^{-1}\right) \partial_{t} v\right. \\
& \left.-2 t^{-2} \tau v+((n-2)+a) \partial_{t} v\right\rangle,
\end{aligned}
$$

where $\langle$,$\rangle is denoted as an inner product in L^{2}$ space in $(-\infty, \infty) \times S^{n-1}$. We compute each term in the inner product in the last identity. Integration by parts shows that

$$
\begin{aligned}
4\left\langle\partial_{t}^{2} v, \quad 2\left(\tau+2 \tau t^{-1}\right) \partial_{t} v\right\rangle & =4 \iint\left(\tau+2 \tau t^{-1}\right) \partial_{t}\left(\partial_{t} v\right)^{2} d t d \omega \\
& =8 \tau \iint t^{-2}\left|\partial_{t} v\right|^{2} d t d \omega .
\end{aligned}
$$

Using integration by parts twice, we have

$$
\begin{aligned}
4\left\langle\partial_{t}^{2} v, \quad-2 t^{-2} \tau v\right\rangle & =-16 \tau \iint t^{-3} \partial_{t} v v d t d \omega+8 \tau \iint t^{-2}\left(\partial_{t} v\right)^{2} d t d \omega \\
& =-24 \tau \iint t^{-4}|v|^{2} d t d \omega+8 \tau \iint t^{-2}\left|\partial_{t} v\right|^{2} d t d \omega .
\end{aligned}
$$

Integration by parts indicates that

$$
\begin{aligned}
4\left\langle\partial_{t}^{2} v, \quad((n-2)+a) \partial_{t} v\right\rangle & =2 \iint((n-2)+a) \partial_{t}\left|\partial_{t} v\right|^{2} d t d \omega \\
& =-2 \iint a^{\prime}\left|\partial_{t} v\right|^{2} d t d \omega .
\end{aligned}
$$

Continuing the computation in (3.10) gives that

$$
\begin{aligned}
4\left\langle\left(\tau+2 \tau t^{-1}\right)^{2} v, \quad 2\left(\tau+2 \tau t^{-1}\right) \partial_{t} v\right\rangle & =4 \iint\left(\tau+2 \tau t^{-1}\right)^{3} \partial_{t} v^{2} d t d \omega \\
& =24 \tau \iint\left(\tau+2 \tau t^{-1}\right)^{2} t^{-2} v^{2} d t d \omega
\end{aligned}
$$


It is clear that

$$
4\left\langle\left(\tau+2 \tau t^{-1}\right)^{2} v, \quad-2 t^{-2} \tau v\right\rangle=-8 \tau \iint\left(\tau+2 \tau t^{-1}\right)^{2} t^{-2} v^{2} d t d \omega .
$$

From integrations by parts, we get

$$
\begin{aligned}
4\left\langle\left(\tau+2 \tau t^{-1}\right)^{2} v, \quad((n-2)+a) \partial_{t} v\right\rangle & =2 \iint((n-2)+a)\left(\tau+2 \tau t^{-1}\right)^{2} \partial_{t} v^{2} d t d \omega \\
& =8 \tau \iint((n-2)+a)\left(\tau+2 \tau t^{-1}\right) t^{-2} v^{2} d t d \omega \\
& -2 \iint a^{\prime}\left(\tau+2 \tau t^{-1}\right)^{2} v^{2} d t d \omega .
\end{aligned}
$$

Similar argument yields that

$$
\begin{aligned}
& 4\left\langle\left[(n-2)\left(\tau+2 \tau t^{-1}\right)+b\right] v, \quad 2\left(\tau+2 \tau t^{-1}\right) \partial_{t} v\right\rangle \\
& =4 \iint\left[(n-2)\left(\tau+2 \tau t^{-1}\right)+b\right]\left(\tau+2 \tau t^{-1}\right) \partial_{t} v^{2} d t d \omega \\
& =-4 \iint\left[-2(n-2) \tau t^{-2}+b^{\prime}\right]\left(\tau+2 \tau t^{-1}\right) v^{2} d t d \omega \\
& +8 \tau \iint\left[(n-2)\left(\tau+2 \tau t^{-1}\right)+b\right] t^{-2} v^{2} d t d \omega .
\end{aligned}
$$

It is obvious that

$$
\begin{aligned}
& 4\left\langle\left[(n-2)\left(\tau+2 \tau t^{-1}\right)+b\right] v, \quad-2 t^{-2} \tau v\right\rangle \\
& =-8 \tau \iint\left[(n-2)\left(\tau+2 \tau t^{-1}\right)+b\right] t^{-2} v^{2} d t d \omega .
\end{aligned}
$$

It follows from integration by parts that

$$
\begin{aligned}
& 4\left\langle\left[(n-2)\left(\tau+2 \tau t^{-1}\right)+b\right] v, \quad((n-2)+a) \partial_{t} v\right\rangle \\
& =2 \iint\left[(n-2)\left(\tau+2 \tau t^{-1}\right)+b\right]((n-2)+a) \partial_{t} v^{2} d t d \omega \\
& =-2 \iint\left(-2(n-2) t^{-2} \tau+b^{\prime}\right)((n-2)+a) v^{2} d t d \omega \\
& -2 \iint\left[(n-2)\left(\tau+2 \tau t^{-1}\right)+b\right] a^{\prime} v^{2} d t d \omega .
\end{aligned}
$$

Since $\triangle_{\omega} v=\sum_{j=1}^{n} \Omega_{j}^{2} v$, integration by parts leads to

$$
\begin{aligned}
4\left\langle\triangle_{\omega} v, \quad 2\left(\tau+2 \tau t^{-1}\right) \partial_{t} v\right\rangle & =-4 \sum_{j=1}^{n} \iint\left(\tau+2 \tau t^{-1}\right) \partial_{t}\left|\Omega_{j} v\right|^{2} d t d \omega \\
& =-8 \tau \sum_{j=1}^{n} \iint t^{-2}\left|\Omega_{j} v\right|^{2} d t d \omega .
\end{aligned}
$$

It follows that

$$
4\left\langle\triangle_{\omega} v, \quad-2 \tau t^{-2} v\right\rangle=8 \tau \sum_{j=1}^{n} \iint t^{-2}\left|\Omega_{j} v\right|^{2} d t d \omega .
$$


It is true from integration by parts that

$$
\begin{aligned}
4\left\langle\triangle_{\omega} v, \quad((n-2)+a) \partial_{t} v\right\rangle & =-4 \sum_{j=1}^{n} \iint((n-2)+a) \Omega_{j} v \Omega_{j} \partial_{t} v d t d \omega \\
& =2 \sum_{j=1}^{n} \iint a^{\prime}\left|\Omega_{j} v\right|^{2} d t d \omega .
\end{aligned}
$$

Taking into account of the equalities from (3.11) to (3.22) gives that

$$
I \geq \iint 15 \tau^{3} t^{-2} v^{2}+\tau^{2} O\left(t^{-2}\right) d t d \omega+15 \tau \iint t^{-2}\left|\partial_{t} v\right|^{2} d t d \omega+2 \sum_{j=1}^{n} \iint a^{\prime}\left|\Omega_{j} v\right|^{2} d t d \omega
$$

for $-\infty<t<t_{0}<0$ with $\left|t_{0}\right|$ large enough.

On the other hand, we consider the integration of the sum of $\triangle_{\tau} v$ and $\triangle_{\tau}^{-} v$. Define

$$
J:=\iint t^{-2}\left|\triangle_{\tau} v\right|^{2} d t d \omega+\iint t^{-2}\left|\triangle_{\tau}^{-} v\right|^{2} d t d \omega .
$$

Direction computations from (3.7) and (3.8) yields that

$$
\begin{aligned}
J= & 2 \iint t^{-2}\left\{\left|\partial_{t}^{2} v\right|^{2}+4\left(\tau+2 \tau t^{-1}\right)^{2}\left|\partial_{t} v\right|^{2}+4 t^{-4} \tau^{2} v^{2}+\left(\tau+2 \tau t^{-1}\right)^{4} v^{2}\right. \\
& \left.+((n-2)+a)^{2}\left|\partial_{t} v\right|^{2}+\left[(n-2)\left(\tau+2 \tau t^{-1}\right)+b\right]^{2} v^{2}+\left|\triangle_{\omega} v\right|^{2}\right\} d t d \omega \\
& +4\left\langle t^{-2} \partial_{t}^{2} v, \quad\left(\tau+2 \tau t^{-1}\right)^{2} v+\left[(n-2)\left(\tau+2 \tau t^{-1}\right)+b\right] v+\triangle_{\omega} v\right\rangle \\
& +4\left\langle t^{-2}\left(\tau+2 \tau t^{-1}\right)^{2} v, \quad\left[(n-2)\left(\tau+2 \tau t^{-1}\right)+b\right] v+\triangle_{\omega} v\right\rangle \\
& +4\left\langle t^{-2}\left[(n-2)\left(\tau+2 \tau t^{-1}\right)+b\right] v, \quad \triangle_{\omega} v\right\rangle \\
& +8\left\langle t^{-2}\left(\tau+2 \tau t^{-1}\right) \partial_{t} v, \quad-2 t^{-2} \tau v+((n-2)+a) v\right\rangle \\
& +4\left\langle-2 t^{-4} \tau v, \quad((n-2)+a) \partial_{t} v\right\rangle .
\end{aligned}
$$

We compute each inner product in the expression of $J$, respectively. Integration by parts shows that

$$
\begin{aligned}
& 4<t^{-2} \partial_{t}^{2} v, \quad\left(\tau+2 \tau t^{-1}\right)^{2} v+\left[(n-2)\left(\tau+2 \tau t^{-1}\right)+b\right] v> \\
& =-4 \tau^{2} \iint t^{-2}\left|\partial_{t} v\right|^{2} d t d \omega+\tau^{2} \iint O\left(t^{-3}\right)\left|\partial_{t} v\right|^{2} d t d \omega+\tau \iint O\left(t^{-2}\right)\left|\partial_{t} v\right|^{2} d t d \omega \\
& +12 \tau^{2} \iint O\left(t^{-4}\right) v^{2} d t d \omega+\tau \iint O\left(t^{-4}\right) v^{2} d t d \omega+\tau^{2} \iint O\left(t^{-5}\right) v^{2} d t d \omega \\
& -4 \iint b t^{-2}\left|\partial_{t} v\right|^{2} d t d \omega+2 \iint \partial_{t}^{2}\left(t^{-2} b\right) v^{2} d t d \omega
\end{aligned}
$$

Again, it follows from integration by parts that

$$
\begin{aligned}
4\left\langle t^{-2} \partial_{t}^{2} v, \quad \triangle_{\omega} v\right\rangle & =4 \sum_{j=1}^{n} \iint t^{-2}\left|\partial_{t} \Omega_{j} v\right|^{2} d t d \omega-8 \iint t^{-3} \partial_{t} \Omega_{j} v \Omega_{j} v d t d \omega \\
& =4 \sum_{j=1}^{n} \iint t^{-2}\left|\partial_{t} \Omega_{j} v\right|^{2} d t d \omega-24 \iint t^{-4}\left|\Omega_{j} u\right|^{2} d t d \omega
\end{aligned}
$$


Direct calculations indicate that

$$
\begin{aligned}
& 4\left\langle t^{-2}\left(\tau+2 \tau t^{-1}\right)^{2} v, \quad\left[(n-2)\left(\tau+2 \tau t^{-1}\right)+b\right] v+\triangle_{\omega} v\right\rangle \\
& =4(n-2) \iint t^{-2}\left(\tau+2 \tau t^{-1}\right)^{3} v^{2} d t d \omega \\
& +4 \iint t^{-2}\left(\tau+2 \tau t^{-1}\right) b v^{2} d t d \omega \\
& -4 \sum_{j=1}^{n} \iint t^{-2}\left(\tau+2 \tau t^{-1}\right)^{2}\left|\Omega_{j} v\right|^{2} d t d \omega .
\end{aligned}
$$

From the integration by parts, we obtain

$$
\begin{aligned}
& 4\left\langle t^{-2}\left[(n-2)\left(\tau+2 \tau t^{-1}\right)+b\right] v, \quad \triangle_{\omega} v\right\rangle \\
& =-4 \sum_{j=1}^{n} \iint t^{-2}\left[(n-2)\left(\tau+2 \tau t^{-1}\right)+b\right]\left|\Omega_{j} v\right|^{2} d t d \omega .
\end{aligned}
$$

It follows that

$$
\begin{aligned}
& 8\left\langle t^{-2}\left(\tau+2 \tau t^{-1}\right) \partial_{t} v, \quad-2 t^{-2} \tau v+((n-2)+a) \partial_{t} v\right\rangle \\
& =\tau^{2} \iint\left(-32 t^{-5}-80 t^{-6}\right) v^{2} d t d \omega \\
& +8 \iint t^{-2}\left(\tau+2 \tau t^{-1}\right)((n-2)+a)\left|\partial_{t} v\right|^{2} d t d \omega
\end{aligned}
$$

Integration by parts yields that

$$
\begin{aligned}
& 4\left\langle-2 t^{-4} \tau v, \quad((n-2)+a) \partial_{t} v\right\rangle \\
& =-4 \iint t^{-4}((n-2)+a) \partial_{t} v^{2} d t d \omega \\
& =-16 \iint t^{-5}((n-2)+a) v^{2} d t d \omega+4 \iint t^{-4} a^{\prime} v^{2} d t d \omega
\end{aligned}
$$

Taking the identities from (3.25) to (3.31) and $\left|t_{0}\right|$ is sufficiently large into consideration gives that

$$
\begin{aligned}
J \geq & 2 \iint t^{-2}\left|\partial_{t}^{2} v\right|^{2} d t d \omega+\iint\left(3 \tau t^{-2}+\tau^{2} O\left(t^{-3}\right)\right)\left|\partial_{t} v\right|^{2} d t d \omega \\
& +2 \sum_{j=1}^{n} \iint t^{-2}\left|\triangle_{\omega} v\right|^{2} d t d \omega+4 \sum_{j=1}^{n} \iint t^{-2}\left|\partial_{t} \Omega_{j} v\right| d t d \omega \\
& +\iint\left(\tau^{4} t^{-2}+\tau^{4} O\left(t^{-3}\right)\right)|v|^{2} d t d \omega \\
& -\sum_{j=1}^{n} \iint\left(5 \tau^{2} t^{-2}+\tau^{2} O\left(t^{-3}\right)\right)\left|\Omega_{j} v\right|^{2} d t d \omega .
\end{aligned}
$$

Now we consider the combined effect from $I$ and $J$, i.e. $\tau I+J$. Using the fact that $-\infty<$ $t<-\left|t_{0}\right|$ with $\left|t_{0}\right|$ large enough and combining the estimates for $I$ in (3.23) and estimates for $J$ 
in (3.32) yields that

$$
\begin{aligned}
\tau I+J & \geq 15 \tau^{4} \iint t^{-2}|v|^{2} d t d \omega+17 \tau^{2} \iint t^{-2}\left|\partial_{t} v\right|^{2} d t d \omega-6 \tau^{2} \sum_{j=1}^{n} \iint t^{-2}\left|\Omega_{j} v\right|^{2} d t d \omega \\
& +2 \iint t^{-2}\left|\partial_{t}^{2} v\right|^{2} d t d \omega+\sum_{j=1}^{n} \iint 2 t^{-2}\left|\triangle_{\omega} v\right|^{2} d t d \omega+4 \sum_{j=1}^{n} \iint t^{-2}\left|\partial_{t} \Omega_{j} v\right|^{2} d t d \omega \\
& =U+\frac{11}{4} \tau^{4} \iint t^{-2}|v|^{2} d t d \omega+17 \tau^{2} \iint t^{-2}\left|\partial_{t} v\right|^{2} d t d \omega \\
& +\tau^{2} \sum_{j=1}^{n} \iint t^{-2}\left|\Omega_{j} v\right|^{2} d t d \omega+2 \iint t^{-2}\left|\partial_{t}^{2} v\right|^{2} d t d \omega+\iint t^{-2}\left|\triangle_{\omega} v\right|^{2} d t d \omega \\
& +4 \sum_{j=1}^{n} \iint t^{-2}\left|\partial_{t} \Omega_{j} v\right|^{2} d t d \omega
\end{aligned}
$$

where

$$
U=\frac{49}{4} \iint t^{-2} \tau^{4}|v|^{2} d t d \omega-7 \tau^{2} \sum_{j=1}^{n} \iint t^{-2}\left|\Omega_{j} v\right|^{2} d t d \omega+\sum_{j=1}^{n} \iint t^{-2}\left|\triangle_{\omega} v\right|^{2} d t d \omega
$$

Note from integration by parts that

$$
-\tau^{2} \sum_{j=1}^{n} \iint t^{-2}\left|\Omega_{j} v\right|^{2} d t d \omega=\tau^{2} \iint t^{-2} \triangle_{\omega} v v d t d \omega
$$

Thus, $U \geq 0$ by Cauchy-Schwartz inequality.

By the ellipticity of $\triangle_{\omega}$, there exists a constant $C$ such that

$$
\sum_{|\alpha|=2} \iint t^{-2}\left|\Omega^{\alpha} v\right|^{2} d t d \omega \leq C \iint t^{-2}\left|\triangle_{\omega} v\right|^{2} d t d \omega
$$

It follows from (3.33) that

$$
\begin{aligned}
C(\tau I+J) & \geq \tau^{4} \iint t^{-2}|v|^{2} d t d \omega+\tau^{2} \iint t^{-2}\left|\partial_{t} v\right|^{2} d t d \omega+\tau^{2} \sum_{j=1}^{n} \iint t^{-2}\left|\Omega_{j} v\right|^{2} d t d \omega \\
& +\iint t^{-2}\left|\partial_{t}^{2} v\right|^{2} d t d \omega+\sum_{|\alpha|=2} \iint t^{-2}\left|\Omega^{\alpha} v\right|^{2} d t d \omega \\
& +\sum_{j=1}^{n} \iint t^{-2}\left|\partial_{t} \Omega_{j} v\right|^{2} d t d \omega
\end{aligned}
$$

Since

$$
\begin{aligned}
(\tau I+J) & =\tau\left\|\triangle_{\tau} v\right\|_{L^{2}}^{2}-\tau\left\|\triangle_{\tau}^{-} v\right\|_{L^{2}}^{2}+\left\|t^{-1} \triangle_{\tau} v\right\|_{L^{2}}^{2}+\left\|t^{-1} \triangle_{\tau}^{-} v\right\|_{L^{2}}^{2} \\
& \leq(\tau+1)\left\|\triangle_{\tau} v\right\|_{L^{2}}^{2}
\end{aligned}
$$


then

$$
\begin{aligned}
(\tau+1)\left\|\triangle_{\tau} v\right\|^{2} & \geq \tau^{4} \iint t^{-2}|v|^{2} d t d \omega+\tau^{2} \iint t^{-2}\left|\partial_{t} v\right|^{2} d t d \omega+\tau^{2} \sum_{j=1}^{n} \iint t^{-2}\left|\Omega_{j} v\right|^{2} d t d \omega \\
& +\iint t^{-2}\left|\partial_{t}^{2} v\right|^{2} d t d \omega+\sum_{|\alpha|=2} \iint t^{-2}\left|\Omega^{\alpha} v\right|^{2} d t d \omega \\
& +\sum_{j=1}^{n} \iint t^{-2}\left|\partial_{t} \Omega_{j} v\right|^{2} d t d \omega
\end{aligned}
$$

That is,

$$
\left\|\triangle_{\tau} v\right\|_{L^{2}}^{2} \geq C \sum_{j+|\alpha| \leq 2} \tau^{3-2(j+|\alpha|)}\left\|t^{-1} \partial_{t}^{j} \Omega^{\alpha} v\right\|_{L^{2}}^{2} .
$$

Returning to the coordinate $(r, \omega)$ and $u$, the latter Carleman estimate (3.38) is equivalent to the following

$$
\begin{aligned}
& C \sum_{|\alpha| \leq 2} \tau^{\frac{3-2|\alpha|}{2}}\left\|e^{-\tau \phi(r)}(\log r)^{\sigma_{2}-1} r^{\sigma_{1}+|\alpha|}\left|D^{\alpha} u\right|\right\|_{L^{2}\left(r^{-n} d x\right)} \\
& \leq\left\|e^{-\tau \phi(r)}(\log r)^{\sigma_{2}} r^{\sigma_{1}+2} \triangle u\right\|_{L^{2}\left(r^{-n} d x\right)} .
\end{aligned}
$$

To get the Carleman estimates for higher order elliptic operators, we iterate the estimate (3.39). Let us consider $m=2$ for example. From (3.39), we obtain

$$
\begin{aligned}
& \left\|e^{-\tau \phi(r)}(\log r)^{\sigma_{2}} r^{\sigma_{1}+4} \triangle^{2} u\right\|_{L^{2}\left(r^{-n} d x\right)} \\
& \geq C \sum_{\left|\alpha_{1}\right| \leq 2} \tau^{\frac{3-2\left|\alpha_{1}\right|}{2}}\left\|e^{-\tau \phi(r)}(\log r)^{\sigma_{2}-1} r^{\sigma_{1}+2+\left|\alpha_{1}\right|}\left|\triangle D^{\alpha_{1}} u\right|\right\|_{L^{2}\left(r^{-n} d x\right)} \\
& \geq C \sum_{\left|\alpha_{1}\right| \leq 2} \sum_{\left|\alpha_{2}\right| \leq 2} \tau^{\frac{3-2\left|\alpha_{1}\right|}{2}} \tau^{\frac{3-2\left|\alpha_{2}\right|}{2}}\left\|e^{-\tau \phi(r)}(\log r)^{\sigma_{2}-2} r^{\sigma_{1}+\left|\alpha_{1}\right|+\left|\alpha_{2}\right|}\left|D^{\alpha_{1}+\alpha_{2}} u\right|\right\|_{L^{2}\left(r^{-n} d x\right)} \\
& \left.=C \sum_{|\alpha| \leq 4} \tau^{\frac{6-2|\alpha|}{2}}\left\|e^{-\tau \phi(r)}(\log r)^{\sigma_{2}-2} r^{\sigma_{1}+|\alpha|}\left|D^{\alpha} u\right|\right\|_{L^{2}\left(r^{-n}\right.} d x\right)
\end{aligned}
$$

for $\alpha=\alpha_{1}+\alpha_{2}$. Thus, the Carleman estimates (3.2) in the case of $m=2$ is shown. Note that $\sigma_{1}$ and $\sigma_{2}$ are defined to be integers. In each iteration, $\sigma_{1}$ and $\sigma_{2}$ represent different integers, which is also the reason the iteration is able to be carried out. Iterating the estimate (3.39) $\mathrm{m}$ times, it implies that

$$
\begin{aligned}
& C \sum_{|\alpha| \leq 2 m} \tau^{\frac{3 m-2|\alpha|}{2}}\left\|e^{-\tau \phi(r)}(\log r)^{\sigma_{2}-m} r^{\sigma_{1}+|\alpha|}\left|D^{\alpha} u\right|\right\|_{L^{2}\left(r^{-n} d x\right)} \\
& \leq\left\|e^{-\tau \phi(r)}(\log r)^{\sigma_{2}} r^{\sigma_{1}+2 m} \triangle^{m} u\right\|_{L^{2}\left(r^{-n} d x\right)} .
\end{aligned}
$$

Therefore, we complete the proof of Proposition 1.

Based on the quantitative $L^{2}$ type Carleman estimates in Proposition 1, we are going to establish a $L^{2} \rightarrow L^{p}$ type Carleman estimates to deal with singular weight potentials. The idea is to use Sobolev embedding and an interpolation argument inspired by the idea in [DZ17].

Proof of Theorem [3. In particular, when $\sigma_{1}=\sigma_{2}=0$, the Carleman estimates (3.2) in Proposition 1 takes the form

$$
\sum_{|\alpha|=0}^{2 m-1} \tau^{\frac{3 m-2|\alpha|}{2}}\left\|e^{-\tau \phi(r)}(\log r)^{-m} r^{|\alpha|}\left|D^{\alpha} u\right|\right\|_{L^{2}\left(r^{-n} d x\right)} \leq\left\|e^{-\tau \phi(r)} r^{2 m} \triangle^{m} u\right\|_{L^{2}\left(r^{-n} d x\right)}
$$


Case I): Now we focus on the norm that only involves $u$. For the case of $n>4 m-2$, since $u \in C_{0}^{\infty}\left(\mathbb{B}_{R}\left(x_{0}\right) \backslash\left\{x_{0}\right\}\right)$, by Sobolev embedding $W^{2 m-1,2} \hookrightarrow L^{\frac{2 n}{n-2(2 m-1)}}$, we obtain the following

$$
\begin{aligned}
\left\|e^{-\tau \phi(r)}(\log r)^{-m} u\right\|_{L^{\frac{2 n}{n-2(2 m-1)}}\left(r^{-n} d x\right)} & \left\|e^{-\tau \phi(r)}(\log r)^{-m} r^{-\frac{n-2(2 m-1)}{2}} u\right\|_{L^{\frac{2 n}{n-2(2 m-1)}}} \\
\leq & C\left\|D^{2 m-1}\left[e^{-\tau \phi(r)}(\log r)^{-m} r^{-\frac{n-2(2 m-1)}{2}} u\right]\right\|_{L^{2}} \\
= & C \sum_{\left|\alpha_{1}\right|+\left|\alpha_{2}\right|+\left|\alpha_{3}\right|+\left|\alpha_{4}\right|=2 m-1} \tau^{\left|\alpha_{4}\right|} \| e^{-\tau \phi(r)} r^{-\left|\alpha_{4}\right|}(\log r)^{-m-\left|\alpha_{3}\right|} r^{-\left|\alpha_{3}\right|} \\
\times & r^{-\frac{n-2(2 m-1)}{2}-\left|\alpha_{2}\right|}\left|D^{\alpha_{1}} u\right| \|_{L^{2}} \\
\leq & \sum_{\left|\alpha_{1}\right|+\left|\alpha_{4}\right| \leq 2 m-1} \tau^{\left|\alpha_{4}\right|}\left\|e^{-\tau \phi(r)} r^{-\frac{n}{2}+\left|\alpha_{1}\right|}(\log r)^{-m}\left|D^{\alpha_{1}} u\right|\right\|_{L^{2}} \\
= & \left.\sum_{\left|\alpha_{1}\right|+\left|\alpha_{4}\right| \leq 2 m-1} \tau^{\left|\alpha_{4}\right|} \tau^{\frac{-3 m+2\left|\alpha_{1}\right|}{2}}\left\|e^{-\tau \phi(r)}(\log r)^{-m} r^{2 m} \triangle^{m} u\right\|_{L^{2}\left(r^{-n}\right.} d x\right) \\
\leq & \left.C \tau^{\frac{m}{2}-1}\left\|e^{-\tau \phi(r)} r^{2 m} \triangle^{m} u\right\|_{L^{2}\left(r^{-n}\right.} d x\right),
\end{aligned}
$$

where we have used that $\phi^{\prime}(r)=\frac{1}{r}+\frac{2}{r \log r} \leq \frac{1}{r}$ since $r \leq R_{0} \leq 1$ and (3.42). It is known from (3.2) that

$$
\left\|e^{-\tau \phi(r)}(\log r)^{-m} u\right\|_{L^{2}\left(r^{-n} d x\right)} \leq C \tau^{\frac{-3 m}{2}}\left\|e^{-\tau \phi(r)} r^{2 m} \triangle^{m} u\right\|_{L^{2}\left(r^{-n} d x\right)} .
$$

We are going to do an interpolation argument with the last two inequalities. Choose $\lambda \in(0,1)$ so that $p=2 \lambda+(1-\lambda) \frac{2 n}{n-4 m+2}$. By Hölder's inequality,

$$
\left\|e^{-\tau \phi(r)}(\log r)^{-m} u\right\|_{L^{p}\left(r^{-n} d x\right)} \leq\left\|e^{-\tau \phi(r)}(\log r)^{-m} u\right\|_{L^{2}\left(r^{-n} d x\right)}^{\frac{2 \lambda}{p}}\left\|e^{-\tau \phi(r)}(\log r)^{-m} u\right\|_{L^{\frac{2 n}{n-4 m+2}\left(r^{-n} d x\right)}}^{\frac{2 n(1-\lambda)}{(n-4 m+2}} .
$$

Since $\lambda=\frac{2 n-p(n-4 m+2)}{8 m-4}$, if we set $\theta=\frac{2 \lambda}{p}$, then

$$
\theta=\frac{2}{p} \cdot \frac{2 n-p(n-4 m+2)}{8 m-4}=\frac{2 n-p(n-4 m+2)}{(4 m-2) p},
$$

and $1-\theta=\frac{(p-2) n}{(4 m-2) p}$. Therefore, from (3.43) and (3.44),

$$
\begin{aligned}
& \left\|e^{-\tau \phi(r)}(\log r)^{-m} u\right\|_{L^{p}\left(r^{-n} d x\right)} \\
& \leq\left\|e^{-\tau \phi(r)}(\log r)^{-m} u\right\|_{L^{2}\left(r^{-n} d x\right)}^{\theta}\left\|e^{-\tau \phi(r)}(\log r)^{-m} u\right\|_{\left.L^{\frac{2 n}{n-4 m+2}\left(r^{-n}\right.} d x\right)}^{1-\theta} \\
& \leq\left[C \tau^{\frac{-3 m}{2}}\left\|e^{-\tau \phi(r)} r^{2 m} \triangle^{m} u\right\|_{L^{2}\left(r^{-n} d x\right)}\right]^{\theta}\left[C \tau^{\frac{m-2}{2}}\left\|e^{-\tau \phi(r)} r^{2 m} \triangle^{m} u\right\|_{L^{2}\left(r^{-n} d x\right)}\right]^{1-\theta} \\
& =C \tau^{-\frac{3 m}{2} \theta+\frac{m-2}{2}(1-\theta)}\left\|e^{-\tau \phi(r)} r^{2 m} \triangle^{m} u\right\|_{L^{2}\left(r^{-n} d x\right)} \\
& =C \tau^{\frac{m-2}{2}-\frac{2 n-p(n-4 m+2)}{2 p}}\left\|e^{-\tau \phi(r)} r^{2 m} \triangle^{m} u\right\|_{L^{2}\left(r^{-n} d x\right)} .
\end{aligned}
$$

That is, for any $2 \leq p \leq \frac{2 n}{n-4 m+2}$, we have

$$
\tau^{\frac{3 m}{2}-\frac{n(p-2)}{2 p}}\left\|e^{-\tau \phi(r)}(\log r)^{-m} u\right\|_{L^{p}\left(r^{-n} d x\right)} \leq C\left\|e^{-\tau \phi(r)} r^{2 m} \triangle^{m} u\right\|_{L^{2}\left(r^{-n} d x\right)} .
$$

It is obvious from (3.42) that

$$
\sum_{|\alpha|=1}^{2 m-1} \tau^{\frac{3 m-2 \alpha}{2}}\left\|e^{-\tau \phi(r)}(\log r)^{-m} r^{|\alpha|} D^{\alpha} u\right\|_{L^{2}\left(r^{-n} d x\right)} \leq C\left\|e^{-\tau \phi(r)} r^{2 m} \triangle^{m} u \mid\right\|_{L^{2}\left(r^{-n} d x\right)}
$$


The combination of the previous two inequalities yields that

$$
\begin{aligned}
\tau^{\frac{3 m}{2}-\frac{n(p-2)}{2 p}}\left\|(\log r)^{-m} e^{-\tau \phi(r)} u\right\|_{L^{p}\left(r^{-n} d x\right)} & +\sum_{|\alpha|=1}^{2 m-1} \tau^{\frac{3 m-2 \alpha}{2}}\left\|e^{-\tau \phi(r)}(\log r)^{-m} r^{|\alpha|}\left|D^{\alpha} u\right|\right\|_{L^{2}\left(r^{-n} d x\right)} \\
& \leq C\left\|e^{-\tau \phi(r)} r^{2 m}\left|\triangle^{m} u\right|\right\|_{L^{2}\left(r^{-n} d x\right)} .
\end{aligned}
$$

This completes the proof of (2.1).

Case II): For the case $n=4 m-2$, we use the same idea as before. By Sobolev embedding $W^{2 m-1,2} \hookrightarrow L^{p^{\prime}}$ for $2 \leq p^{\prime}<\infty$, we obtain that

$$
\begin{aligned}
& \left\|e^{-\tau \phi(r)}(\log r)^{-m} u\right\|_{L^{p^{\prime}}} \\
& \leq C\left\|D^{2 m-1}\left[e^{-\tau \phi(r)}(\log r)^{-m} u\right]\right\|_{L^{2}} \\
& =C \sum_{\left|\alpha_{1}\right|+\left|\alpha_{2}\right|+\left|\alpha_{3}\right|+\left|\alpha_{4}\right|=2 m-1} \tau^{\left|\alpha_{1}\right|}\left\|e^{-\tau \phi(r)} r^{-\left|\alpha_{1}\right|}(\log r)^{-m-\left|\alpha_{2}\right|} r^{-\left|\alpha_{2}\right|-\left|\alpha_{4}\right|} D^{\alpha_{3}} u\right\|_{L^{2}} \\
& \leq C \sum_{\left|\alpha_{1}\right|+\left|\alpha_{2}\right|+\left|\alpha_{3}\right| \leq 2 m-1} \tau^{\left|\alpha_{1}\right|}\left\|e^{-\tau \phi(r)} r^{-(2 m-1)+\frac{n}{2}}(\log r)^{-m-\left|\alpha_{2}\right|} r^{\left|\alpha_{3}\right|} D^{\alpha_{3}} u\right\|_{L^{2}\left(r^{-n} d x\right)} \\
& \leq C \sum_{\left|\alpha_{1}\right|+\left|\alpha_{3}\right| \leq 2 m-1} \tau^{\left|\alpha_{1}\right|}\left\|e^{-\tau \phi(r)}(\log r)^{-m} r^{\left|\alpha_{3}\right|} D^{\alpha_{3}} u\right\|_{L^{2}\left(r^{-n} d x\right)} \\
& \leq C \sum_{\left|\alpha_{1}\right|+\left|\alpha_{3}\right| \leq 2 m-1} \tau^{\left|\alpha_{1}\right|} \tau^{\frac{-3 m+2\left|\alpha_{3}\right|}{2}}\left\|e^{-\tau \phi(r)} r^{2 m} \triangle^{m} u\right\|_{L^{2}\left(r^{-n} d x\right)} \\
& \left.\leq C \tau^{\frac{m}{2}-1}\left\|e^{-\tau \phi(r)} r^{2 m} \triangle^{m} u\right\|_{L^{2}\left(r^{-n}\right.} d x\right),
\end{aligned}
$$

where we have used the fact $n=4 m-2$ and (3.42). From (3.2), we have

$$
\left\|e^{-\tau \phi(r)}(\log r)^{-m} u\right\|_{L^{2}} \leq C \tau^{\frac{-3 m}{2}}\left\|e^{-\tau \phi(r)} r^{2 m} \triangle^{m} u \mid\right\|_{L^{2}\left(r^{-n} d x\right)} .
$$

As before, we interpolate the last two inequalities. Choose $\lambda \in(0,1)$ so that $p=2 \lambda+(1-\lambda) p^{\prime}$. Note that $2<p<p^{\prime}$. The Hölder's inequality implies that

$$
\left\|e^{-\tau \phi(r)}(\log r)^{-m} u\right\|_{L^{p}} \leq\left\|e^{-\tau \phi(r)}(\log r)^{-m} u\right\|_{L^{2}}^{\frac{2 \lambda}{p}}\left\|e^{-\tau \phi(r)}(\log r)^{-m} u\right\|_{L^{p^{\prime}}}^{\frac{p^{\prime}(1-\lambda)}{p}} .
$$

Since $\lambda=\frac{p^{\prime}-p}{p^{\prime}-2}$, if we set $\theta=\frac{2 \lambda}{p}=\frac{2\left(p^{\prime}-p\right)}{p\left(p^{\prime}-2\right)}$, then

$$
1-\theta=1-\frac{2\left(p^{\prime}-p\right)}{p\left(p^{\prime}-2\right)}=\frac{p^{\prime}(p-2)}{\left(p^{\prime}-2\right) p}
$$

and we have that $0 \leq \theta \leq 1$. Therefore,

$$
\begin{aligned}
& \left\|e^{-\tau \phi(r)}(\log r)^{-m} u\right\|_{L^{p}} \\
& \leq\left\|e^{-\tau \phi(r)}(\log r)^{-m} u\right\|_{L^{2}}^{\theta}\left\|e^{-\tau \phi(r)}(\log r)^{-m} u\right\|_{L^{p^{\prime}}}^{1-\theta} \\
& \leq\left[C \tau^{\frac{-3 m}{2}}\left\|e^{-\tau \phi(r)} r^{2 m} \triangle^{m} u\right\|_{L^{2}\left(r^{-n} d x\right)}\right]^{\theta}\left[C \tau^{\frac{m-2}{2}}\left\|e^{-\tau \phi(r)} r^{2 m} \triangle^{m} u\right\|_{L^{2}\left(r^{-n} d x\right)}\right]^{1-\theta} \\
& =C \tau^{\frac{m-2}{2}-\frac{(4 m-2) \theta}{2}}\left\|e^{-\tau \phi(r)} r^{2 m} \triangle^{m} u\right\|_{L^{2}\left(r^{-n} d x\right)} \\
& =C \tau^{\frac{m-2}{2}-\frac{(4 m-2)}{2} \frac{2\left(p^{\prime}-p\right)}{p\left(p^{\prime}-2\right)}}\left\|e^{-\tau \phi(r)} r^{2 m} \triangle^{m} u\right\|_{L^{2}\left(r^{-n} d x\right)} \\
& =C \tau^{\frac{m-2}{2}-\frac{(4 m-2)}{p}(1-\varepsilon)}\left\|e^{-\tau \phi(r)} r^{2 m} \triangle^{m} u\right\|_{L^{2}\left(r^{-n} d x\right)},
\end{aligned}
$$

where we have used (3.48) and (3.49). Here $\epsilon=\frac{p-2}{p^{\prime}-2}$. Since $p<p^{\prime}<\infty$, then $0<\epsilon<1$. Moreover, $p^{\prime}$ can be any sufficiently large constant that approaches to infinity so that $\epsilon$ can be 
chosen to be any sufficiently small constant that approaches 0 . Thus, for any $2<p<\infty$,

$$
\tau^{\frac{4 m-2}{p}(1-\varepsilon)-\frac{m-2}{2}}\left\|(\log r)^{-m} e^{-\tau \phi(r)} u\right\|_{L^{p}} \leq C\left\|e^{-\tau \phi(r)} r^{2 m} \triangle^{m} u\right\|_{L^{2}\left(r^{-n} d x\right)} .
$$

Combing (3.42) with the last inequality gives the proof of (2.2) in Theorem 3 ,

Case III): For the case of $2 \leq n<4 m-2$, by the Sobolev embedding inequality, $W^{2 m-1,2} \hookrightarrow$ $L^{\infty}$, we have

$$
\left\|e^{-\tau \phi(r)}(\log r)^{-m} u\right\|_{L^{\infty}} \leq C\left\|D^{2 m-1}\left[e^{-\tau \phi(r)}(\log r)^{-m} u\right]\right\|_{L^{2}} .
$$

The similar arguments as Case I and II shows that

$$
\left\|e^{-\tau \phi(r)}(\log r)^{-m} u\right\|_{L^{\infty}} \leq C \tau^{\frac{m}{2}-1}\left\|e^{-\tau \phi(r)} r^{2 m} \triangle^{m} u\right\|_{L^{2}\left(r^{-n} d x\right)} .
$$

The estimates (2.3) gives that

$$
\left\|e^{-\tau \phi(r)}(\log r)^{-m} u\right\|_{L^{2}} \leq C \tau^{\frac{-3 m}{2}}\left\|e^{-\tau \phi(r)} r^{2 m} \triangle^{m} u \mid\right\|_{L^{2}\left(r^{-n} d x\right)} .
$$

Using Hölder's inequality, for any $2 \leq p \leq \infty$, we interpolate the inequality (3.51) and (3.52),

$$
\begin{aligned}
& \left\|e^{-\tau \phi(r)}(\log r)^{-m} u\right\|_{L^{p}} \\
& \leq\left\|e^{-\tau \phi(r)}(\log r)^{-m} u\right\|_{L^{2}}^{\frac{2}{p}}\left\|e^{-\tau \phi(r)}(\log r)^{-m} u\right\|_{L^{\infty}}^{1-\frac{2}{p}} \\
& \leq\left[C \tau^{\frac{-3 m}{2}}\left\|e^{-\tau \phi(r)} r^{2 m} \triangle^{m} u\right\|_{L^{2}\left(r^{-n} d x\right)}\right]^{\frac{2}{p}}\left[C \tau^{\frac{m-2}{2}}\left\|e^{-\tau \phi(r)} r^{2 m} \triangle^{m} u\right\|_{L^{2}\left(r^{-n} d x\right)}\right]^{1-\frac{2}{p}} \\
& \leq \tau^{\frac{m-2}{2}-\frac{(4 m-2)}{p}}\left\|e^{-\tau \phi(r)} r^{2 m} \triangle^{m} u\right\|_{L^{2}\left(r^{-n} d x\right)} .
\end{aligned}
$$

Together (3.42) with the last inequality gives the proof of (2.3) in Theorem 3 .

Finally, we arrive at the proof of the three cases in Theorem 3 .

\section{VANISHING ORDER}

In this section, we show the vanishing order of the solutions. For the preparations, we need some kind of Caccioppoli inequality and a $L^{\infty}$ bound estimate. We first prove a quantitative type Caccioppoli inequality for the higher order elliptic equation. In such inequality, we want to know how the coefficient depends on the norms of the potential $V_{0}$ and coefficient functions $V_{\alpha}$. The idea is adapted from the Corollary 17.1.4 in the classical book of Hörmander [H85]. More delicate analysis is required to take care of appearance of singular potential $V_{0}$.

Lemma 1. Let $u$ be the solution in (1.1). There exists a positive constant $C$ that does not depend of $u$ such that

$$
\sum_{|\alpha|=0}^{2 m-1}\left\|r^{|\alpha|} D^{\alpha} u\right\|_{L^{2}\left(\mathbb{B}_{c_{2} R_{0}} \backslash \mathbb{B}_{c_{3} R_{0}}\right)} \leq C\left(\sum_{|\alpha|=1}^{\alpha_{0}}\left\|V_{\alpha}\right\|_{L^{\infty}}+\left\|V_{0}\right\|_{L^{s}}+1\right)^{2 m-1}\|u\|_{L^{2}\left(\mathbb{B}_{c_{1} R_{0}} \backslash \mathbb{B}_{c_{4} R_{0}}\right)}
$$

for all positive constants $0<c_{4}<c_{3}<c_{2}<c_{1}$.

Proof. From Corollary 17.3 in [H85], it holds that

$$
\left\|d^{|\alpha|}(x) D^{\alpha} u\right\|_{L^{2}(X)} \leq C\left(\left\|d^{2 m}(x) \triangle^{m} u\right\|_{L^{2}(X)}+\|u\|_{L^{2}(X)}\right)^{\frac{|\alpha|}{2 m}\|u\|_{L^{2}(X)}^{1-\frac{|\alpha|}{2 m}},}
$$

where $d(x)$ is the distance from $X$ to $X^{c}$ and $X^{c}$ is the complement of $X$. We apply the estimate (4.2) with $X=\mathbb{B}_{c_{1} R_{0}} \backslash \mathbb{B}_{c_{4} R_{0}}$ for some small $R_{0}$ with $0<c_{4}<c_{1}$. Since $u$ is the solution of the 
equation (1.1),

$$
\begin{aligned}
d^{2 m} \triangle^{m} u & =d^{2 m}\left(\sum_{|\alpha|=1}^{\alpha_{0}} V_{\alpha} D^{\alpha} u+V_{0} u\right) \\
& =\sum_{|\alpha|=1}^{\alpha_{0}} d^{2 m-|\alpha|}(x) d^{|\alpha|}(x) V_{\alpha} D^{\alpha} u+d^{2 m} V_{0} u
\end{aligned}
$$

From Hölder's inequality, it follows that

$$
\left\|d^{2 m}(x) V_{0} u\right\|_{L^{2}(X)} \leq\left\|V_{0}\right\|_{L^{s}(X)}\left\|d^{2 m}(x) u\right\|_{L^{\frac{2 s}{s-2}}(X)} \cdot
$$

We first consider the case $n>4 m-2$. Since $\frac{n}{2 m-1} \leq \frac{2 n}{3 m}<s \leq \infty$, then $2 \leq \frac{2 s}{s-2}<\frac{2 n}{2 n-3 m} \leq$ $\frac{2 n}{n-4 m+2}$. Note that $d(x)$ is a smooth function if $R_{0}$ is small. From the Sobolev embedding $W^{2 m-1,2} \hookrightarrow L^{\frac{2 n}{n-2(2 m-1)}}$, we obtain

$$
\left\|d^{2 m} u\right\|_{L^{\frac{2 s}{s-2}(X)}} \leq C\left(\sum_{|\alpha|=2 m-1}\left\|D^{\alpha}\left(d^{2 m} u\right)\right\|_{L^{2}(X)}+\left\|d^{2 m} u\right\|_{L^{2}(X)}\right) .
$$

In the case of $n=4 m-2$, since $s>\frac{4(2 m-1)}{3 m}$, then $s>2$. From the Sobolev embedding $W^{2 m-1,2} \hookrightarrow L^{p}$, for any $2 \leq p<\infty$, we can also obtain (4.5) with a different constant $C$ that does not depend on $u$. It follows from (4.4) and (4.5) that

$$
\begin{aligned}
\left\|d^{2 m}(x) V_{0} u\right\|_{L^{2}(X)} & \leq C\left\|V_{0}\right\|_{L^{s}(X)}\left(\sum_{|\alpha|=0}^{2 m-1}\left\|d^{1+|\alpha|} D^{\alpha} u\right\|_{L^{2}(X)}+\left\|d^{2 m} u\right\|_{L^{2}(X)}\right) \\
& \leq C\left\|V_{0}\right\|_{L^{s}(X)}\left(\sum_{|\alpha|=0}^{2 m-1}\left\|d^{1+|\alpha|} D^{\alpha} u\right\|_{L^{2}(X)}\right)
\end{aligned}
$$

In the case $2 \leq n<4 m-2$, similar arguments as before show that

$$
\left\|d^{2 m}(x) V_{0} u\right\|_{L^{2}(X)} \leq C\left\|V_{0}\right\|_{L^{s}(X)}\left(\sum_{|\alpha|=0}^{2 m-1}\left\|d^{1+|\alpha|} D^{\alpha} u\right\|_{L^{2}(X)}\right) .
$$

Choose

$$
\beta=\sum_{|\alpha|=1}^{2 m-1}\left\|V_{\alpha}\right\|_{L^{\infty}}+\left\|V_{0}\right\|_{L^{s}}+1 .
$$

Using the estimates (4.2) and (4.3), it follows that

$$
\begin{aligned}
\left\|d^{|\alpha|}(x) D^{\alpha} u\right\|_{L^{2}(X)} & \leq C\left(\sum_{|\alpha|=1}^{2 m-1} R_{0}^{2 m-\alpha}\left\|V_{\alpha}\right\|_{L^{\infty}}\left\|d^{|\alpha|}(x) D^{\alpha} u\right\|_{L^{2}(X)}\right. \\
& \left.+\left\|d^{2 m} V_{0} u\right\|_{L^{2}(X)}+\|u\|_{L^{2}(X)}\right)^{\frac{|\alpha|}{2 m}}\|u\|_{L^{2}(X)}^{1-\frac{|\alpha|}{2 m}} \\
& \leq C\left(\beta \sum_{|\alpha|=0}^{2 m-1}\left\|d^{|\alpha|}(x) D^{\alpha} u\right\|_{L^{2}(X)}\right)^{\frac{|\alpha|}{2 m}\|u\|_{L^{2}(X)}^{1-\frac{|\alpha|}{2 m}}},
\end{aligned}
$$

where we applied the estimate (4.6) for the case $n \geq 4 m-2$ and (4.7) for the case $2 \leq n<4 m-2$ in last inequality, and the fact that $R_{0}$ is small. Let

$$
S=\sum_{|\alpha|=0}^{2 m-1}\left\|d^{|\alpha|}(x) D^{\alpha} u\right\|_{L^{2}(X)} .
$$


Taking the sum for $0 \leq \alpha \leq 2 m-1$, from (4.8), we obtain

$$
\begin{aligned}
S & \leq C \sum_{|\alpha| \leq 2 m-1}(\beta S)^{\frac{|\alpha|}{2 m}\|u\|_{L^{2}(X)}^{1-\frac{|\alpha|}{2 m}}} \\
& \leq C(\beta S)^{\frac{2 m-1}{2 m}}\|u\|_{L^{2}(X)}^{\frac{1}{2 m}} .
\end{aligned}
$$

Thus,

$$
S \leq C \beta^{2 m-1}\|u\|_{L^{2}(X)} .
$$

From (4.10), we have

$$
\sum_{|\alpha|=0}^{2 m-1} \int_{c_{2} R_{0} \leq|d(x)| \leq c_{3} R_{0}}\left|d^{|\alpha|}(x) D^{\alpha} u\right|^{2} d x \leq C \beta^{4 m-2} \int_{c_{4} R_{0} \leq|d(x)| \leq c_{1} R_{0}}|u|^{2} d x
$$

for all $0<c_{4}<c_{3}<c_{2}<c_{1}$. This completes the proof of the lemma.

We need to establish a $L^{\infty}$-version of three-ball theorem. However, it seems that the classical De Giorgi-Nash-Moser theory does not exist for higher order elliptic equations. We will deduce the estimate by Sobolev embedding and a $W^{2 m, p}$ type estimate. We first present a $W^{2 m, p}$ type estimates for higher order elliptic equations (see e.g. [ADN59]). Let $u$ satisfy the following equation

$$
\triangle^{m} u+\sum_{|\alpha|=0}^{2 m-1} V_{\alpha} D^{\alpha} u=g(x) \quad \text { in } \mathbb{B}_{1},
$$

where $\left\|V_{\alpha}\right\|_{L^{\infty}} \leq 1$ for every $\alpha$. Then we have

Lemma 2. Let $1<p<\infty$. Suppose $u \in W^{2 m, p}$ satisfies $\sqrt{4.12)}$. Then there exits a constant $C>0$ depending only on $n, m$ such that for any $\sigma \in(0,1)$,

$$
\|u\|_{W^{2 m, p}\left(\mathbb{B}_{\sigma}\right)} \leq \frac{C(n, m)}{(1-\sigma)^{2 m}}\left(\|g\|_{L^{p}\left(\mathbb{B}_{1}\right)}+\|u\|_{L^{p}\left(\mathbb{B}_{1}\right)}\right) .
$$

We are going to establish a $L^{\infty}$ bound for the solution of

$$
\triangle^{m} u+\sum_{|\alpha|=1}^{\alpha_{0}} V_{\alpha}(x) \cdot D^{\alpha} u+V_{0}(x) u=0
$$

using the last lemma.

Lemma 3. Let $u$ be the solution in (1.1). There exists a positive constant $C$ independent of $u$ such that

$$
\|u\|_{L^{\infty}\left(\mathbb{B}_{r}\right)} \leq C\left(\sum_{|\alpha|=1}^{\alpha_{0}}\left\|V_{\alpha}\right\|_{L^{\infty}}+\left\|V_{0}\right\|_{L^{s}}+1\right)^{\frac{n}{2}} r^{-\frac{n}{2}}\|u\|_{L^{2}\left(\mathbb{B}_{2 r}\right)} .
$$

Proof. We do a scaling argument. Let $w(x)=u(R r x)$, where

$$
R=\frac{1}{C\left(\sum_{|\alpha|=1}^{\alpha_{0}}\left\|V_{\alpha}\right\|_{L^{\infty}}+\left\|V_{0}\right\|_{L^{s}}^{\frac{s}{2 m s-n}}+1\right)}
$$

and $0<r<1$. Then $w(x)$ satisfies the following equation

$$
\triangle^{m} w+\sum_{|\alpha|=1}^{\alpha_{0}} \tilde{V}_{\alpha} D^{\alpha} w+\tilde{V}_{0} w=0
$$


where

$$
\tilde{V}_{\alpha}=R^{2 m-\alpha} r^{2 m-\alpha} V_{\alpha}(R r x) \quad \text { and } \quad \tilde{V}_{0}=R^{2 m} r^{2 m} V_{0}(R r x) .
$$

It is easy to check that $\left\|\tilde{V}_{\alpha}\right\|_{L^{\infty}} \leq 1$ and $\left\|\tilde{V}_{0}\right\|_{L^{s}} \leq 1$. We use the $W^{2 m, p}$ estimate to get the $L^{\infty}$ bound, then use the scaling argument to find that how the coefficients depend on the norm of $V_{\alpha}$ and $V_{0}$.

We first study the case $n>4 m-2$ and $s>\frac{2 n}{3 m}$ for higher order elliptic equations. Assume that $g(x)$ is $\tilde{V}_{0} w$ in Lemma 2 . By the $W^{2 m, p}$ estimate in Lemma 2 with $p=\frac{2 s}{s+2}$ and Hölder's inequality with $\frac{s+2}{2 s}=\frac{1}{s}+\frac{1}{2}$, we obtain

$$
\begin{aligned}
\|w\|_{W^{2 m, \frac{2 s}{s+2}\left(\mathbb{B}_{\sigma}\right)}} \leq C(\sigma)\left[\|w\|_{L^{\frac{2 s}{s+2}\left(\mathbb{B}_{1}\right)}}+\left\|\tilde{V}_{0} w\right\|_{L^{\frac{2 s}{s+2}\left(\mathbb{B}_{1}\right)}}\right] \\
\leq C(\sigma)\left(1+\left\|\tilde{V}_{0}\right\|_{L^{s}}\right)\|w\|_{L^{2}\left(\mathbb{B}_{1}\right)} \\
\leq C(\sigma)\|w\|_{L^{2}\left(\mathbb{B}_{1}\right)} .
\end{aligned}
$$

By Sobolev embedding $W^{2 m, \frac{2 s}{s+2}} \hookrightarrow L^{\frac{2 n s}{n(s+2)-4 m s}}$, it follows that

$$
\|w\|_{L^{\frac{2 n s}{(n-4 m) s+2 n}\left(\mathbb{B}_{\sigma}\right)}} \leq C(\sigma)\|w\|_{L^{2}\left(\mathbb{B}_{1}\right)} .
$$

Obviously, $w$ in a smaller Lebesgue $L^{p}$ space with $p=\frac{2 n s}{(n-4 m) s+2 n}>2$.

Using the $W^{2 m, p}$ estimate in Lemma 2 again with $p=\frac{2 n s}{(n-4 m) s+4 n}$ and Hölder's inequality with $\frac{1}{p}=\frac{1}{s}+\frac{(n-4 m) s+2 n}{2 n s}$, we get

$$
\begin{aligned}
\|w\|_{W^{2 m, \frac{2 n s}{(n-4 m) s+4 n}}\left(\mathbb{B}_{\sigma^{2}}\right)} \leq C(\sigma)\left[\|w\|_{L^{\frac{2 n s}{(n-4 m) s+4 n}}\left(\mathbb{B}_{\sigma}\right)}+\left\|\tilde{V}_{0} w\right\|_{L^{\frac{2 n s}{(n-4 m) s+4 n}}\left(\mathbb{B}_{1}\right)}\right] \\
\leq C(\sigma)\left(1+\left\|\tilde{V}_{0}\right\|_{L^{s}}\right)\|w\|_{L^{\frac{2 n s}{(n-4 m) s+2 n}}\left(\mathbb{B}_{\sigma}\right)} \\
\leq C(\sigma)\|w\|_{L^{2}\left(\mathbb{B}_{1}\right) .}
\end{aligned}
$$

By Sobolev embedding, it can be deduced that

$$
\|w\|_{W^{2 m, \frac{2 n s}{(n-4 m-4) s+4 n}}\left(\mathbb{B}_{\sigma^{2}}\right)} \leq C(\sigma)\|w\|_{L^{2}\left(\mathbb{B}_{1}\right)} .
$$

If we continue this argument as performed before, we will get that $u$ in a smaller $L^{p}$ space with a larger exponent $p$. Assume that we have obtained

$$
\|w\|_{L^{\frac{q s}{s-q}}\left(\mathbb{B}_{\sigma^{k-1}}\right)} \leq C(\sigma)\|w\|_{L^{2}\left(\mathbb{B}_{1}\right)}
$$

for some $q$ very close to $\frac{n}{2 m}$ after $k-1$ steps. Furthermore, the $W^{2 m, p}$ estimate with $p=q$ and Hölder's inequality with $\frac{1}{q}=\frac{1}{s}+\frac{s-q}{s q}$ yields that

$$
\begin{aligned}
\|w\|_{W^{2 m, q}\left(\mathbb{B}_{\sigma^{k}}\right)} & \leq C(\sigma)\left[\|w\|_{L^{q}\left(\mathbb{B}_{\sigma^{k}}\right)}+\left\|\tilde{V}_{0} w\right\|_{L^{q}\left(\mathbb{B}_{\sigma^{k}}\right)}\right] \\
& \leq C(\sigma)\|w\|_{L^{\frac{q s}{s-q}}\left(\mathbb{B}_{\sigma^{k}}\right)} \\
& \leq C(\sigma)\|w\|_{L^{2}\left(\mathbb{B}_{1}\right)} .
\end{aligned}
$$

Keep in mind that $s>\frac{2 n}{3 m}$. We choose some $q^{\prime}$ such that $\frac{n}{2 m}<q^{\prime}<\frac{2 n}{3 m}$. Since $q$ is very close to $\frac{n}{2 m}$, by Sobolev embedding $W^{2 m, q} \hookrightarrow L^{\frac{n q}{n-2 m q}}$ with $\frac{n q}{n-2 m q}>\frac{q^{\prime} s}{s-q^{\prime}}$, we can have that

$$
\|w\|_{L^{\frac{q^{\prime} s}{s-q^{\prime}}\left(\mathbb{B}_{\sigma^{k}}\right)}} \leq C(\sigma)\|w\|_{L^{2}\left(\mathbb{B}_{1}\right)}
$$


for some $k$ depending on $n, m$ and $s$. Using the $W^{2 m, p}$ estimate with $p=q^{\prime}$ and Hölder's inequality again, we have

$$
\begin{aligned}
& \|w\|_{W^{2 m, q^{\prime}}\left(\mathbb{B}_{\sigma^{k+1}}\right)} \leq C(\sigma)\left[\|w\|_{L^{q^{\prime}\left(\mathbb{B}_{\sigma^{k}}\right)}}+\left\|\tilde{V}_{0} w\right\|_{L^{q^{\prime}\left(\mathbb{B}_{\sigma^{k}}\right)}}\right] \\
& \leq C(\sigma)\|w\|_{L^{\frac{q^{\prime} s}{s-q^{\prime}}}\left(\mathbb{B}_{\sigma^{k}}\right)} \\
& \leq C(\sigma)\|w\|_{L^{2}\left(\mathbb{B}_{1}\right)} \text {. }
\end{aligned}
$$

The Sobolev embedding implies that

$$
\|w\|_{L^{\infty}\left(\mathbb{B}_{\sigma^{k+1}}\right)} \leq C(\sigma)\|w\|_{W^{2 m, q^{\prime}\left(\mathbb{B}_{\sigma^{k+1}}\right)}} \leq C(\sigma)\|w\|_{L^{2}\left(\mathbb{B}_{1}\right)} .
$$

Let $\sigma^{k+1}=\frac{1}{2}$. Recall that $w(x)=u(R r x)$, the latter inequality implies

$$
\|u\|_{L^{\infty}\left(\mathbb{B}_{R r / 2}\right)} \leq C(R r)^{-\frac{n}{2}}\|u\|_{L^{2}\left(\mathbb{B}_{R r}\right)} .
$$

If $2 \leq n \leq 4 m-2$, we can carry out the similar argument to get the $L^{\infty}$ bound (4.22). Actually, it takes fewer iterations.

For any $\mathbb{B}_{r}$, assume that the maximum value for $|u|$ is achieved in $\mathbb{B}_{r}$ at $x_{0}$. That is, $\|u\|_{L^{\infty}\left(\mathbb{B}_{r}\right)}=\left|u\left(x_{0}\right)\right|$. Using the inequality (4.22) at the ball $\mathbb{B}_{R r}\left(x_{0}\right)$ yields that

$$
\begin{aligned}
\|u\|_{L^{\infty}\left(\mathbb{B}_{r}\right)} & \leq C(R r)^{-\frac{n}{2}}\|u\|_{L^{2}\left(\mathbb{B}_{R r}\left(x_{0}\right)\right)} \\
& \leq C(R r)^{-\frac{n}{2}}\|u\|_{L^{2}\left(\mathbb{B}_{2 r}\right)}
\end{aligned}
$$

Considering the definition of $R$, we obtain

$$
\|u\|_{L^{\infty}\left(\mathbb{B}_{r}\right)} \leq C\left(\sum_{|\alpha|=1}^{\alpha_{0}}\left\|V_{\alpha}\right\|_{L^{\infty}}+\left\|V_{0}\right\|_{L^{s}}^{\frac{s}{2 m s-n}}+1\right)^{\frac{n}{2}} r^{-\frac{n}{2}}\|u\|_{L^{2}\left(\mathbb{B}_{2 r}\right)} .
$$

Since $\frac{s}{2 m s-n} \leq 1$ if and only if $s \geq \frac{n}{2 m-1}$, we can check $\frac{s}{2 m s-n} \leq 1$ from the assumption of $s$ in all cases $n>4 m-2, n=4 m-2$ and $2 \leq n<4 m-2$. Thus, the estimate (4.14) is achieved.

Therefore, we arrive at (4.14) in the lemma in those three cases.

Using the new $L^{2} \rightarrow L^{p}$ type Carleman estimate in Theorem 4 for higher order elliptic equations, we establish a three-ball inequality that plays an important role in obtaining the vanishing order. The three-ball inequality is also considered as a quantitative behavior of the strong unique continuation property. The argument is motivated by those in [Ken07].

Lemma 4. Let $0<r_{0}<r_{1}<R_{1}<R_{0}$, where $R_{0}<1$ is sufficiently small. Let $u$ be a solution to (1.1) in $B_{R_{0}}$.

I): In the case of $n>4 m-2$, assume that $s \in\left(\frac{2 n}{3 m}, \infty\right]$, then

$$
\begin{aligned}
& \|u\|_{L^{\infty}\left(\mathbb{B}_{3 r_{1} / 4}\right)} \leq C \beta^{2 m+\frac{n}{2}}\left|\log r_{1}\right|^{m}\left[\|u\|_{L^{\infty}\left(\mathbb{B}_{2 r_{0}}\right)}\right]^{k_{0}}\left[\|u\|_{L^{\infty}\left(\mathbb{B}_{R_{1}}\right)}\right]^{1-k_{0}} \\
& +C \beta^{\frac{n}{2}}\left(\frac{R_{1}}{r_{1}}\right)^{\frac{n}{2}} \exp \left[C\left(1+\sum_{|\alpha|=1}^{\alpha_{0}}\left\|V_{\alpha}\right\|_{L^{\infty}}^{\mu}+\left\|V_{0}\right\|_{L^{s}}^{\nu}\right)\left(\phi\left(\frac{R_{1}}{2}\right)-\phi\left(r_{0}\right)\right)\right]\|u\|_{L^{\infty}\left(\mathbb{B}_{2 r_{0}}\right)},
\end{aligned}
$$

where $k_{0}=\frac{\phi\left(\frac{R_{1}}{2}\right)-\phi\left(r_{1}\right)}{\phi\left(\frac{R_{1}}{2}\right)-\phi\left(r_{0}\right)}, \beta=C\left(1+\sum_{|\alpha|=1}^{\alpha_{0}}\left\|V_{\alpha}\right\|_{L^{\infty}}+\left\|V_{0}\right\|_{L^{s}}\right)$, and $\mu$ and $\nu$ are as given in Theorem 4. 
II): In the case of $n=4 m-2$, assume that $s \in\left(\frac{4(2 m-1)}{3 m}, \infty\right]$, then

$$
\begin{aligned}
& \|u\|_{L^{\infty}\left(\mathbb{B}_{3 r_{1} / 4}\right)} \leq C \beta^{2 m+\frac{n}{2}}\left|\log r_{1}\right|^{m}\left[\|u\|_{L^{\infty}\left(\mathbb{B}_{2 r_{0}}\right)}\right]^{k_{0}}\left[\|u\|_{L^{\infty}\left(\mathbb{B}_{R_{1}}\right)}\right]^{1-k_{0}} \\
& +C \beta^{\frac{n}{2}}\left(\frac{R_{1}}{r_{1}}\right)^{\frac{n}{2}} \exp \left[C\left(1+\sum_{|\alpha|=1}^{\alpha_{0}}\left\|V_{\alpha}\right\|_{L^{\infty}}^{\mu}+\left\|V_{0}\right\|_{L^{s}}^{\tilde{v}}\right)\left(\phi\left(\frac{R_{1}}{2}\right)-\phi\left(r_{0}\right)\right)\right]\|u\|_{L^{\infty}\left(\mathbb{B}_{2 r_{0}}\right)},
\end{aligned}
$$

where $k_{0}$ and $\beta$ are the same as those in Case $I$, and $\mu$ and $\tilde{\nu}$ are as given in Theorem 4 .

III): In the case of $2 \leq n<4 m-2$, assume that $s \in\left(\frac{4(2 m-1)}{3 m}, \infty\right]$ then

$$
\begin{aligned}
& \|u\|_{L^{\infty}\left(\mathbb{B}_{3 r_{1} / 4}\right)} \leq C \beta^{2 m+\frac{n}{2}}\left|\log r_{1}\right|^{m}\left[\|u\|_{L^{\infty}\left(\mathbb{B}_{2 r_{0}}\right)}\right]^{k_{0}}\left[\|u\|_{L^{\infty}\left(\mathbb{B}_{R_{1}}\right)}\right]^{1-k_{0}} \\
& +C \beta^{\frac{n}{2}}\left(\frac{R_{1}}{r_{1}}\right)^{\frac{n}{2}} \exp \left[C\left(1+\sum_{|\alpha|=1}^{\alpha_{0}}\left\|V_{\alpha}\right\|_{L^{\infty}}^{\mu}+\left\|V_{0}\right\|_{L^{s}}^{\bar{\nu}}\right)\left(\phi\left(\frac{R_{1}}{2}\right)-\phi\left(r_{0}\right)\right)\right]\|u\|_{L^{\infty}\left(\mathbb{B}_{2 r_{0}}\right)},
\end{aligned}
$$

where $k_{0}$ and $\beta$ are the same as those in Case $I$ and II, and $\mu$ and $\bar{\nu}$ are as given in Theorem 4 .

Proof. We first consider the case $n>4 m-2$ and $s \in\left(\frac{2 n}{3 m}, \infty\right]$. Let $r_{0}<r_{1}<R_{1}$. The standard notation $[a, b]$ is denoted as closed annulus with inner radius $a$ and outer radius $b$. Choose a smooth function $\eta \in C_{0}^{\infty}\left(\mathbb{B}_{R_{0}}\right)$ with $B_{2 R_{1}} \subset B_{R_{0}}$. Let

$$
D_{1}=\left[\frac{3}{2} r_{0}, \frac{1}{2} R_{1}\right], \quad D_{2}=\left[r_{0}, \frac{3}{2} r_{0}\right], \quad D_{3}=\left[\frac{1}{2} R_{1}, \frac{3}{4} R_{1}\right] .
$$

We define $\eta$ as $\eta=1$ on $D_{1}$ and $\eta=0$ on $\left[0, r_{0}\right] \cup\left[\frac{3}{4} R_{1}, R_{1}\right]$. Then we have $\left|D^{\alpha} \eta\right| \leq \frac{C}{r_{0}^{|\alpha|}}$ on $D_{2}$. Similarly, $\left|D^{\alpha} \eta\right| \leq \frac{C}{R_{1}^{|\alpha|}}$ on $D_{3}$.

Since $u$ is a solution to (1.1) in $\mathbb{B}_{R_{0}}$, by regularity argument mentioned in the introduction, $u \in L^{\infty}\left(\mathbb{B}_{R_{1}}\right) \cap W^{2 m, 2}\left(\mathbb{B}_{R_{1}}\right)$. Therefore, by regularization, the estimate in Theorem 4 holds for $\eta u$. To use the Carleman estimates in Theorem 4, we substitute $\eta u$ into (2.4). The following holds

$$
\begin{aligned}
& \tau^{\beta_{0}}\left\|e^{-\tau \phi(r)}(\log r)^{-m} \eta u\right\|_{L^{p}\left(r^{-n} d x\right)}+\sum_{|\alpha|=1}^{2 m-1} \tau^{\beta_{\alpha}}\left\|e^{-\tau \phi(r)}(\log r)^{-m} r^{|\alpha|} D^{\alpha} u\right\|_{L^{2}\left(r^{-n} d x\right)} \\
& \leq C\left\|e^{-\tau \phi(r)} r^{2 m}\left(\triangle^{m}(\eta u)+\sum_{|\alpha|=1}^{\alpha_{0}} V_{\alpha} D^{\alpha}(\eta u)+V_{0} \eta u\right)\right\|_{L^{2}\left(r^{-n} d x\right)},
\end{aligned}
$$

whenever

$$
\tau>C\left(1+\sum_{|\alpha|=1}^{\alpha_{0}}\left\|V_{\alpha}\right\|_{L^{\infty}}^{\mu}+\left\|V_{0}\right\|_{L^{p}}^{\nu}\right) .
$$

Consider that $u$ is a solution to equation (1.1), further calculations show that

$$
\begin{aligned}
& \tau^{\beta_{0}}\left\|e^{-\tau \phi(r)}(\log r)^{-m} \eta u\right\|_{L^{p}\left(r^{-n} d x\right)} \\
& \leq C\left\|e^{-\tau \phi(r)} r^{2 m}\left(\left[\triangle^{m}, \eta\right] u+\eta \triangle^{m} u+\sum_{|\alpha|=1}^{\alpha_{0}} V_{\alpha}\left[D^{\alpha}, \eta\right] u+\sum_{|\alpha|=1}^{\alpha_{0}} \eta D^{\alpha} u+V_{0} \eta u\right)\right\|_{L^{2}\left(r^{-n} d x\right)} \\
& \leq C \| e^{-\tau \phi(r)} r^{2 m}\left(\left[\triangle^{m}, \eta\right] u+\sum_{|\alpha|=1}^{\alpha_{0}} V_{\alpha}\left[D^{\alpha}, \eta\right] u \|_{L^{2}\left(r^{-n} d x\right)}\right.
\end{aligned}
$$


Note that $\left[\triangle^{m}, \eta\right]$ is a $2 m-1$ order differential operator on $u$ involving the derivative of $\eta$. From the last inequality and $p \geq 2$, we have

$$
\tau^{\beta_{0}}\left\|e^{-\tau \phi(r)}(\log r)^{-m} \eta u\right\|_{L^{2}\left(r^{-n} d x\right)} \leq C \mathcal{K},
$$

where

$$
\begin{aligned}
\mathcal{K} & =\| e^{-\tau \phi(r)} r^{2 m}\left(\left[\triangle^{m}, \eta\right] u+\sum_{|\alpha|=1}^{\alpha_{0}} V_{\alpha}\left[D^{\alpha}, \eta\right] u \|_{L^{2}\left(r^{-n} d x\right)}\right. \\
& \left.\leq \sum_{\alpha=0}^{2 m-1} C\left\|e^{-\tau \phi(r)} r^{|\alpha|}\left|D^{\alpha} u\right|\right\|_{L^{2}\left(D_{2} \cup D_{3}, r^{-n}\right.} d x\right) \\
& \left.+\sum_{|\alpha|=1}^{\alpha_{0}}\left(\left\|V_{\alpha}\right\|_{L^{\infty}}+1\right)\left\|e^{-\tau \phi(r)} r^{|\alpha|}\left|D^{\alpha} u\right|\right\|_{L^{2}\left(D_{2} \cup D_{3}, r^{-n}\right.} d x\right) .
\end{aligned}
$$

Recall that $\beta=C\left(\sum_{|\alpha|=1}^{\alpha_{0}}\left\|V_{\alpha}\right\|_{L^{\infty}}+\left\|V_{0}\right\|_{L^{s}}+1\right)$. By Lemma 1 and the fact that $-\phi(r)$ is decreasing, we have

$$
\begin{aligned}
\left\|e^{-\tau \phi(r)} r^{|\alpha|} D^{\alpha} u\right\|_{L^{2}\left(D_{2}, r^{-n} d x\right)} & \leq C e^{-\tau \phi\left(r_{0}\right)} r_{0}^{-\frac{n}{2}}\left\|r^{|\alpha|} D^{\alpha} u\right\|_{L^{2}\left(D_{2}, d x\right)} \\
& \leq C \beta^{2 m-1} e^{-\tau \phi\left(r_{0}\right)} r_{0}^{-\frac{n}{2}}\|u\|_{L^{2}\left(\mathbb{B}_{2 r_{0}} \backslash \mathbb{B}_{r_{0} / 2}\right)} .
\end{aligned}
$$

Similarly,

$$
\begin{aligned}
\left\|e^{-\tau \phi(r)} r^{|\alpha|} D^{\alpha} u\right\|_{L^{2}\left(D_{3}, r^{-n} d x\right)} & \leq e^{-\tau \phi\left(\frac{R_{1}}{2}\right)} R_{1}^{-\frac{n}{2}}\left\|r^{|\alpha|} D^{\alpha} u\right\|_{L^{2}\left(D_{3}, d x\right)} \\
& \leq C \beta^{2 m-1} e^{-\tau \phi\left(\frac{R_{1}}{2}\right)} R_{1}^{-\frac{n}{2}}\|u\|_{L^{2}\left(\mathbb{B}_{R_{1}} \backslash \mathbb{B}_{R_{1} / 4}\right)} .
\end{aligned}
$$

We conclude that

$$
\begin{aligned}
\mathcal{K} & \leq C \beta^{2 m} e^{-\tau \phi\left(r_{0}\right)} r_{0}^{-\frac{n}{2}}\|u\|_{L^{2}\left(\mathbb{B}_{2 r_{0}} \backslash \mathbb{B}_{r_{0} / 2}\right)} \\
& +C \beta^{2 m} e^{-\tau \phi\left(\frac{R_{1}}{2}\right)} R_{1}^{-\frac{n}{2}}\|u\|_{L^{2}\left(\mathbb{B}_{R_{1}} \backslash \mathbb{B}_{R_{1} / 4}\right)}
\end{aligned}
$$

Define a new set $D_{4}=\left\{r \in D_{1}, r \leq r_{1}\right\}$. From (4.30) and the fact that $\tau \geq 1$ and $\beta_{0}>0$, it is attained that

$$
\begin{aligned}
\|u\|_{L^{2}\left(D_{4}\right)} & \leq \tau^{\beta_{0}}\|u\|_{L^{2}\left(D_{4}\right)} \\
& \left.\leq \tau^{\beta_{0}}\left\|e^{\tau \phi(r)}(\log r)^{m} r^{\frac{n}{2}}\right\|_{L^{\infty}\left(D_{4}\right)}\left\|e^{-\tau \phi(r)}(\log r)^{-m} u\right\|_{L^{2}\left(D_{4}, r^{-n}\right.} d x\right) \\
& \leq e^{\tau \phi\left(r_{1}\right)}\left|\log r_{1}\right|^{m} r_{1}^{\frac{n}{2}} \mathcal{K},
\end{aligned}
$$

where the fact that $e^{\tau \phi(r)}|\log r|^{m} r^{\frac{n}{2}}$ is increasing on $D_{4}$ for $R_{0}$ sufficiently small is used. Adding $\|u\|_{L^{2}\left(\mathbb{B}_{3 r_{0} / 2}\right)}$ to both sides of the last inequality and taking the upper bound of $\mathcal{K}$ into account yields that

$$
\begin{aligned}
\|u\|_{L^{2}\left(\mathbb{B}_{r_{1}}\right)} & \leq C\left|\log r_{1}\right|^{m} \beta^{2 m}\left(\frac{r_{1}}{r_{0}}\right)^{\frac{n}{2}} e^{\tau\left[\phi\left(r_{1}\right)-\phi\left(r_{0}\right)\right]}\|u\|_{L^{2}\left(\mathbb{B}_{2 r_{0}}\right)} \\
& +C\left|\log r_{1}\right|^{m} \beta^{2 m}\left(\frac{r_{1}}{R_{1}}\right)^{\frac{n}{2}} e^{\tau\left[\phi\left(r_{1}\right)-\phi\left(\frac{R_{1}}{2}\right)\right]}\|u\|_{L^{2}\left(\mathbb{B}_{R_{1}}\right)} .
\end{aligned}
$$


Let $U_{1}=\|u\|_{L^{2}\left(\mathbb{B}_{2 r_{0}}\right)}, U_{2}=\|u\|_{L^{2}\left(\mathbb{B}_{R_{1}}\right)}$ and define

$$
\begin{aligned}
& B_{1}=C\left|\log r_{1}\right|^{m} \beta^{2 m}\left(\frac{r_{1}}{r_{0}}\right)^{\frac{n}{2}}, \\
& B_{2}=C\left|\log r_{1}\right|^{m} \beta^{2 m}\left(\frac{r_{1}}{R_{1}}\right)^{\frac{n}{2}} .
\end{aligned}
$$

Then the last inequality leads to

$$
\|u\|_{L^{2}\left(\mathbb{B}_{r_{1}}\right)} \leq B_{1}\left[\frac{\exp \left(\phi\left(r_{1}\right)\right)}{\exp \left(\phi\left(r_{0}\right)\right)}\right]^{\tau} U_{1}+B_{2}\left[\frac{\exp \left(\phi\left(r_{1}\right)\right)}{\exp \left(\phi\left(\frac{R_{1}}{2}\right)\right)}\right]^{\tau} U_{2} .
$$

Define a new parameter $k_{0}$ as follow

$$
\frac{1}{k_{0}}=\frac{\phi\left(\frac{R_{1}}{2}\right)-\phi\left(r_{0}\right)}{\phi\left(\frac{R_{1}}{2}\right)-\phi\left(r_{1}\right)} .
$$

Recall that $\phi(r)=\log r+\log (\log r)^{2}$. If we fix $r_{1}$ and $R_{1}$, and choose $r_{0}$ to be sufficiently small, i.e. $r_{0} \ll r_{1}$, then $\frac{1}{k_{0}} \simeq \log \frac{1}{r_{0}}$. Set

$$
\tau_{1}=\frac{k_{0}}{\phi\left(\frac{R_{1}}{2}\right)-\phi\left(r_{1}\right)} \log \left(\frac{B_{2} U_{2}}{B_{1} U_{1}}\right) .
$$

If $\tau_{1} \geq C\left(1+\sum_{|\alpha|=1}^{\alpha_{0}}\left\|V_{\alpha}\right\|_{L^{\infty}}^{\mu}+\left\|V_{0}\right\|_{L^{s}}^{\nu}\right)$, then the previous calculations hold with $\tau=\tau_{1}$. We get from (4.34) that

$$
\|u\|_{L^{2}\left(\mathbb{B}_{r_{1}}\right)} \leq 2\left(B_{1} U_{1}\right)^{k_{0}}\left(B_{2} U_{2}\right)^{1-k_{0}} .
$$

On the other hand, if $\tau_{1}<C\left(1+\sum_{\alpha=1}^{\alpha_{0}}\left\|V_{\alpha}\right\|_{L^{\infty}}^{\mu}+\left\|V_{0}\right\|_{L^{s}}^{\nu}\right)$, it follows that

$$
U_{2}<\frac{B_{1}}{B_{2}} \exp \left[C\left(1+\sum_{|\alpha|=1}^{\alpha_{0}}\left\|V_{\alpha}\right\|_{L^{\infty}}^{\mu}+\left\|V_{0}\right\|_{L^{s}}^{\nu}\right)\left(\phi\left(\frac{R_{1}}{2}\right)-\phi\left(r_{0}\right)\right)\right] U_{1} .
$$

We can write the last inequality as

$$
\|u\|_{L^{2}\left(\mathbb{B}_{r_{1}}\right)} \leq C\left(\frac{R_{1}}{r_{0}}\right)^{\frac{n}{2}} e^{C\left(1+\sum_{|\alpha|=1}^{\alpha_{0}}\left\|V_{\alpha}\right\|_{L \infty}^{\mu}+\left\|V_{0}\right\|_{L^{p}}^{\nu}\right)\left(\phi\left(\frac{R_{1}}{2}\right)-\phi\left(r_{0}\right)\right)}\|u\|_{L^{2}\left(\mathbb{B}_{2 r_{0}}\right)} .
$$

Together with (4.35) and (4.36), we obtain that

$$
\begin{aligned}
\|u\|_{L^{2}\left(\mathbb{B}_{r_{1}}\right)} & \leq C\left(\sum_{|\alpha|=1}^{\alpha_{0}}\left\|V_{\alpha}\right\|_{L^{\infty}}+\left\|V_{0}\right\|_{L^{s}}+1\right)^{2 m}\left|\log r_{1}\right|^{m} r_{1}^{\frac{n}{2}}\left[r_{0}^{-\frac{n}{2}}\|u\|_{L^{2}\left(\mathbb{B}_{2 r_{0}}\right)}\right]^{k_{0}} \\
& \times\left[R_{1}^{-\frac{n}{2}}\|u\|_{L^{2}\left(\mathbb{B}_{R_{1}}\right)}\right]^{1-k_{0}} \\
& +C\left(\frac{R_{1}}{r_{0}}\right)^{\frac{n}{2}} e^{C\left(1+\sum_{|\alpha|=1}^{\alpha_{0}}\left\|V_{\alpha}\right\|_{L^{\infty}}^{\mu}+\left\|V_{0}\right\|_{L^{s}}^{\nu}\right)\left(\phi\left(\frac{R_{1}}{2}\right)-\phi\left(r_{0}\right)\right)}\|u\|_{L^{2}\left(\mathbb{B}_{2 r_{0}}\right) .}
\end{aligned}
$$

Recall from Lemma 3 that

$$
\|u\|_{L^{\infty}\left(\mathbb{B}_{r}\right)} \leq C\left(\sum_{|\alpha|=1}^{\alpha_{0}}\left\|V_{\alpha}\right\|_{L^{\infty}}+\left\|V_{0}\right\|_{L^{s}}+1\right)^{\frac{n}{2}} r^{-\frac{n}{2}}\|u\|_{L^{2}\left(\mathbb{B}_{2 r}\right)} .
$$

Combining the estimates (4.37) and (4.38), the three-ball inequality in the $L^{\infty}$-norm in the form of (4.25) is derived.

For the case $n=4 m-2$ and $s \in\left(\frac{4(2 m-1)}{3 m}, \infty\right]$, the same argument using the Carleman estimates (2.5) will give (4.26). If $2 \leq n<4 m-2$ and $s \in\left(\frac{4(2 m-1)}{3 m}, \infty\right]$, we can also obtain the 
inequality (4.27) from the Carleman estimates (2.6) by performing the same argument as Case I. This completes the proof of the lemma.

The inequalities (4.25), (4.26) and (4.27) are the three-ball inequalities we use in the proof of Theorem 1. We first use the three-ball inequality in the propagation of smallness argument to establish a lower bound for the solution on $\mathbb{B}_{r}$. Similar arguments have been performed in [Zhu16]. Then we use the three-ball inequality again to establish the order of vanishing estimate.

Proof of Theorem 1. Without loss of generality, we may assume that $x_{0}$ is the origin. We first consider the case $I$. Let $r_{0}=\frac{r}{2}, r_{1}=4 r$ and $R_{1}=10 r$. Then the estimate (4.25) implies that

$$
\begin{aligned}
\|u\|_{L^{\infty}\left(\mathbb{B}_{3 r}\right)} & \leq C \beta^{\frac{4 m+n}{2}}|\log r|^{m}\|u\|_{L^{\infty}\left(\mathbb{B}_{r}\right)}^{k_{0}}\|u\|_{L^{\infty}\left(\mathbb{B}_{10 r}\right)}^{1-k_{0}} \\
& +C \exp \left[C\left(1+\sum_{|\alpha|=1}^{\alpha_{0}}\left\|V_{\alpha}\right\|_{L^{\infty}}^{\mu}+\left\|V_{0}\right\|_{L^{s}}^{\nu}\right)\left(\phi(5 r)-\phi\left(\frac{r}{2}\right)\right)\right]\|u\|_{L^{\infty}\left(\mathbb{B}_{r}\right)},
\end{aligned}
$$

where $k_{0}=\frac{\phi(5 r)-\phi(4 r)}{\phi(5 r)-\phi\left(\frac{r}{2}\right)}$. It is obvious that

$$
c \leq \phi(5 r)-\phi\left(\frac{r}{2}\right) \leq C \quad \text { and } \quad c \leq \phi(5 r)-\phi(4 r) \leq C,
$$

where $C$ and $c$ are positive constants are independent of $r$. Thus, the parameter $k_{0}$ does not depend on $r$.

We choose a small $r<\frac{1}{2}$ such that

$$
\sup _{\mathbb{B}_{r}(0)}|u|=\delta,
$$

where $\delta>0$. Otherwise, by the unique continuation, $u \equiv 0$ in $\mathbb{B}_{1}$, which is impossible. Since $\sup _{|x| \leq 1}|u(x)| \geq 1$, by continuity, there exists some $\bar{x} \in \mathbb{B}_{1}$ such that $|u(\bar{x})|=\sup _{|x| \leq 1}|u(x)| \geq 1$. There $|x| \leq 1$ also exists a sequence of balls with radius $r$, centered at $x_{0}=0, x_{1}, \ldots, x_{d}$ so that $x_{i+1} \in \mathbb{B}_{r}\left(x_{i}\right)$ for every $i$, and $\bar{x} \in \mathbb{B}_{r}\left(x_{d}\right)$. The number of balls, $d$, depends on the radius $r$ that will be fixed later. The application of $L^{\infty}$-version of three-ball inequality (4.39) at the origin and the boundedness assumption that $\|u\|_{L^{\infty}\left(\mathbb{B}_{10}\right)} \leq \hat{C}$ yield that

$$
\begin{aligned}
\|u\|_{L^{\infty}\left(\mathbb{B}_{3 r}(0)\right)} & \leq C \delta^{k_{0}}\left(\sum_{1 \leq \alpha \leq 2 m-1}\left\|V_{\alpha}\right\|_{L^{\infty}}+\left\|V_{0}\right\|_{L^{s}}+1\right)^{C}|\log r|^{m} \\
& +\delta \exp \left[C\left(1+\sum_{|\alpha|=1}^{\alpha_{0}}\left\|V_{\alpha}\right\|_{L^{\infty}}^{\mu}+\left\|V_{0}\right\|_{L^{s}}^{\nu}\right)\right] .
\end{aligned}
$$

By the way each $\mathbb{B}_{r}\left(x_{i}\right)$ is chosen, we obtain $\mathbb{B}_{r}\left(x_{i+1}\right) \subset \mathbb{B}_{3 r}\left(x_{i}\right)$. Hence, for every $i=1,2, \ldots, d$,

$$
\|u\|_{L^{\infty}\left(\mathbb{B}_{r}\left(x_{i+1}\right)\right)} \leq\|u\|_{L^{\infty}\left(\mathbb{B}_{3 r}\left(x_{i}\right)\right)} .
$$

Repeating the above argument with balls centered at $x_{i}$ and making use of (4.40) give that

$$
\|u\|_{L^{\infty}\left(\mathbb{B}_{3 r}\left(x_{i}\right)\right)} \leq C_{i} \delta^{D_{i}}|\log r|^{F_{i}} \exp \left[H_{i}\left(1+\sum_{|\alpha|=1}^{\alpha_{0}}\left\|V_{\alpha}\right\|_{L^{\infty}}^{\mu}+\left\|V_{0}\right\|_{L^{s}}^{\nu}\right)\right]
$$

for $i=0,1, \cdots, d$, where $C_{i}$ is a constant depending on $d, n, m, s, \hat{C}$, and $C$ from Lemma 4 and $D_{i}, E_{i}, F_{i} H_{i}$ are constants depending on $n, m$, and $d$. By the fact that $|u(\bar{x})| \geq 1$ and 
$\bar{x} \in B_{3 r}\left(x_{d}\right)$, we get

$$
\delta \geq c \exp \left[-C\left(1+\sum_{|\alpha|=1}^{\alpha_{0}}\left\|V_{\alpha}\right\|_{L^{\infty}}^{\mu}+\left\|V_{0}\right\|_{L^{s}}^{\nu}\right)\right]|\log r|^{-C}
$$

where $C$ depends on $d, n, m$, and $\hat{C}$.

Now the radius $r$ is fixed as a small number so that $d$ is a fixed constant. We are going to apply the three-ball inequality again. Let $\frac{3}{4} r_{1}=r, R_{1}=10 r$ and let $r_{0}<<r$, i.e. $r_{0}$ is sufficiently small with respect to $r$. Hence, the three-ball inequality (4.25) implies that

$$
\delta \leq I_{1}+I_{2}
$$

where

$$
\begin{aligned}
& I_{1}=C \beta^{2 m+\frac{n}{2}}|\log r|^{m}\left[\|u\|_{L^{\infty}\left(\mathbb{B}_{2 r_{0}}\right)}\right]^{k_{0}}\left[\|u\|_{L^{\infty}\left(\mathbb{B}_{10 r}\right)}\right]^{1-k_{0}}, \\
& I_{2}=C \beta^{\frac{n}{2}} e^{C\left(1+\sum_{|\alpha|=1}^{\alpha_{0}}\left\|V_{\alpha}\right\|_{L^{\infty}}^{\mu}+\left\|V_{0}\right\|_{L^{s}}^{\nu}\right)\left(\phi(5 r)-\phi\left(r_{0}\right)\right)}\|u\|_{L^{\infty}\left(\mathbb{B}_{2 r_{0}}\right)}
\end{aligned}
$$

with $k_{0}=\frac{\phi(5 r)-\phi\left(\frac{4}{3} r\right)}{\phi(5 r)-\phi\left(r_{0}\right)}$.

If $I_{1} \leq I_{2}$, we have

$$
\begin{aligned}
& \exp \left[-C\left(1+\sum_{|\alpha|=1}^{\alpha_{0}}\left\|V_{\alpha}\right\|_{L^{\infty}}^{\mu}+\left\|V_{0}\right\|_{L^{s}}^{\nu}\right)\right]|\log r|^{-C} \leq \delta \leq 2 I_{2} \\
& \leq 2 \beta^{\frac{n}{2}} e^{C\left(1+\sum_{|\alpha|=1}^{\alpha_{0}}\left\|V_{\alpha}\right\|_{L^{\infty}}^{\mu}+\left\|V_{0}\right\|_{L^{s}}^{\nu}\right)\left(\phi(5 r)-\phi\left(r_{0}\right)\right)}\|u\|_{L^{\infty}\left(\mathbb{B}_{2 r_{0}}\right) .}
\end{aligned}
$$

Since $r_{0}<<r$, it is true that $\phi\left(r_{0}\right)-(C+\phi(5 r)) \geq c \phi\left(r_{0}\right)$. We get that

$$
\|u\|_{L^{\infty}\left(\mathbb{B}_{2 r_{0}}\right)} \geq C r_{0}^{C\left(1+\sum_{|\alpha|=1}^{\alpha_{0}}\left\|V_{\alpha}\right\|_{L^{\infty}}^{\mu}+\left\|V_{0}\right\|_{L^{s}}^{\nu}\right)} .
$$

Instead, if $I_{2} \leq I_{1}$, we obtain that

$$
\begin{aligned}
& \exp \left[-C\left(1+\sum_{|\alpha|=1}^{\alpha_{0}}\left\|V_{\alpha}\right\|_{L^{\infty}}^{\mu}+\left\|V_{0}\right\|_{L^{s}}^{\nu}\right)\right]|\log r|^{-C} \leq \delta \leq 2 I_{1} \\
& \leq 2 C \beta^{2 m+\frac{n}{2}}|\log r|^{m}\left[\|u\|_{L^{\infty}\left(\mathbb{B}_{2 r_{0}}\right)}\right]^{k_{0}}\left[\|u\|_{L^{\infty}\left(\mathbb{B}_{10 r}\right)}\right]^{1-k_{0}} .
\end{aligned}
$$

If we raise both sides to $\frac{1}{k_{0}}$ in the last inequality and take the assumption $\|u\|_{L^{\infty}\left(B_{10 r}\right)} \leq \hat{C}$ into consideration, it follows that

$$
\|u\|_{L^{\infty}\left(\mathbb{B}_{2 r_{0}}\right)} \geq C\left(\frac{C}{\hat{C}|\log r|^{C}}\right)^{\frac{1}{k_{0}}} \exp \left[-\frac{C}{k_{0}}\left(1+\sum_{|\alpha|=1}^{\alpha_{0}}\left\|V_{\alpha}\right\|_{L^{\infty}}^{\mu}+\left\|V_{0}\right\|_{L^{s}}^{\nu}\right)\right] \text {. }
$$

Recall that $\frac{1}{k_{0}} \simeq \log \frac{1}{r_{0}}$ if $r_{0}$ is sufficiently small compared with $r$. We arrive at

$$
\|u\|_{L^{\infty}\left(\mathbb{B}_{2 r_{0}}\right)} \geq C r_{0}^{C\left(1+\sum_{|\alpha|=1}^{\alpha_{0}}\left\|V_{\alpha}\right\|_{L^{\infty}}^{\mu}+\left\|V_{0}\right\|_{L^{s}}^{\nu}\right)}
$$

which shows the proof of case I in Theorem 1.

The case $\Pi$ or III follows from the same argument using the three-ball inequality (4.26) or (4.27). Therefore, the proof of Theorem 1 is done. 


\section{Quantitative unique CONTINUATION at INFinity}

In this section, we show the proof Theorem 2, By the maximal order of vanishing estimates, the quantitative unique continuation at infinity is established using the idea of scaling arguments in $[\mathrm{BK} 05]$.

Proof of Theorem Q 2. Case I): We consider the case $n>4 m-2$ and $s \in\left(\frac{2 n}{3 m}, \infty\right]$. Assume $u$ be a solution to (1.1) in $\mathbb{R}^{n}$. Let $x_{0} \in \mathbb{R}^{n}$ and set $\left|x_{0}\right|=R$. Define $u_{R}(x)=u\left(x_{0}+R x\right)$. Set

$$
V_{\alpha, R}(x)=R^{2 m-|\alpha|} V_{\alpha}\left(x_{0}+R x\right) \quad \text { and } \quad V_{0, R}(x)=R^{2 m} V_{0}\left(x_{0}+R x\right) .
$$

For any $r>0$, elementary calculations show that

$$
\begin{aligned}
\left\|V_{0, R}\right\|_{L^{s}\left(\mathbb{B}_{r}(0)\right)} & =\left(\int_{\mathbb{B}_{r}(0)}\left|R^{2 m} V_{0}\left(x_{0}+R x\right)\right|^{s} d x\right)^{\frac{1}{s}} \\
& =R^{2 m-\frac{n}{s}}\left\|V_{0}\right\|_{L^{s}\left(\mathbb{B}_{r R}\left(x_{0}\right)\right)}
\end{aligned}
$$

Thus,

It is clear that

$$
\left\|V_{0, R}\right\|_{L^{s}\left(\mathbb{B}_{10}(0)\right)} \leq A_{0} R^{2 m-\frac{n}{s}}
$$

$$
\left\|V_{\alpha, R}\right\|_{L^{\infty}\left(\mathbb{B}_{10}(0)\right)}=R^{2 m-\alpha}\left\|V_{\alpha}\right\|_{L^{\infty}\left(\mathbb{B}_{10 R}\left(x_{0}\right)\right)} \leq A_{\alpha} R^{2 m-\alpha} .
$$

We can check that $u_{R}$ satisfies the following scaled version of (1.1) in $\mathbb{B}_{10}$,

$$
\begin{aligned}
& \Delta u_{R}(x)+\sum_{|\alpha|=1}^{\alpha_{0}} V_{\alpha, R}(x) D^{\alpha} u_{R}(x)+V_{0, R}(x) u_{R}(x) \\
& =R^{2 m} \Delta u\left(x_{0}+R x\right)+\sum_{|\alpha|=1}^{\alpha_{0}} R^{2 m-\alpha} V_{\alpha, R}(x) R^{\alpha} D^{\alpha} u_{R}(x) \\
& +R^{2 m} V_{0, R}\left(x_{0}+R x\right) u\left(x_{0}+R x\right) \\
& =0 .
\end{aligned}
$$

Obviously,

$$
\left\|u_{R}\right\|_{L^{\infty}\left(\mathbb{B}_{10}\right)}=\|u\|_{L^{\infty}\left(\mathbb{B}_{10 R}\left(x_{0}\right)\right)} \leq C_{0} .
$$

Set $\widetilde{x_{0}}:=-x_{0} / R$. Then $\left|\widetilde{x_{0}}\right|=1$ and $\left|u_{R}\left(\widetilde{x_{0}}\right)\right|=|u(0)| \geq 1$. Namely, $\left.|| u_{R}\right|_{L^{\infty}\left(B_{1}\right)} \geq 1$. Therefore, if $R>>1$, then the application of Theorem 1 to $u_{R}$ and $\hat{C}=C_{0}$ yields that

$$
\begin{aligned}
\|u\|_{L^{\infty}\left(\mathbb{B}_{1}\left(x_{0}\right)\right)} & =\left\|u_{R}\right\|_{L^{\infty}\left(\mathbb{B}_{1 / R}(0)\right)} \\
& \geq c(1 / R)^{C\left[1+\sum_{|\alpha|=1}^{\alpha_{0}}\left(A_{\alpha} R^{2 m-|\alpha|}\right)^{\mu}+C_{2}\left(A_{0} R^{2 m-\frac{n}{s}}\right)^{\nu}\right]} \\
& =c \exp \left\{-C\left[1+\sum_{|\alpha|=1}^{\alpha_{0}}\left(A_{\alpha} R^{2 m-|\alpha|}\right)^{\mu}+C_{2}\left(A_{0} R^{2 m-\frac{n}{s}}\right)^{\nu}\right] \log R\right\} .
\end{aligned}
$$

Recall that $\mu=\frac{2}{3 m-2|\alpha|}$ and $\nu=\frac{2 s}{3 m s-2 n}$. We can check that $(2 m-|\alpha|) \mu$ is increasing with respect to $|\alpha|$. So its maximum value is achieved at $\frac{2\left(2 m-\alpha_{0}\right)}{3 m-\alpha_{0}}$. It can be shown that

$$
\max \left\{(2 m-|\alpha|) \mu,\left(2 m-\frac{n}{s}\right) \nu\right\}=\Theta:= \begin{cases}\frac{2\left(2 m-\alpha_{0}\right)}{3 m-2 \alpha_{0}} & \alpha_{0} \geq \frac{n}{s} \\ \frac{2(2 m s-n)}{3 m s-2 n} & \alpha_{0}<\frac{n}{s}\end{cases}
$$


Therefore,

$$
\|u\|_{L^{\infty}\left(\mathbb{B}_{1}\left(x_{0}\right)\right)} \geq c \exp \left[-C\left(n, m, s, A_{0}, \cdots, A_{\alpha_{0}}\right) R^{\Theta} \log R\right] .
$$

Case II): In the case of $n=4 m-2$ and $s \in\left(\frac{4(2 m-1)}{3 m}, \infty\right]$, similar arguments work. We need to find the $\max \left\{(2 m-|\alpha|) \mu,\left(2 m-\frac{n}{s}\right) \tilde{\nu}\right\}$. Recall that

$$
\tilde{\nu}=\frac{2 s}{3 m s-4(2 m-1)-2(2 m-1)(s-2) \varepsilon} \text {. }
$$

We can check that

$$
\begin{aligned}
& \max \left\{(2 m-|\alpha|) \mu,\left(2 m-\frac{n}{s}\right) \tilde{\nu}\right\} \\
& =\tilde{\Theta}:= \begin{cases}\frac{2\left(2 m-\alpha_{0}\right)}{3 m-2 \alpha_{0}} & \alpha_{0} \geq \frac{8 m(2 m-1)-3 m n+4 m(2 m-1)(s-2) \epsilon}{m s+4(2 m-1)-2 n+2(2 m-1)(s-2) \epsilon}, \\
\frac{2(2 m s-n)}{3 m s-4(2 m-1)-2(2 m-1)(s-2) \epsilon} & \alpha_{0}<\frac{8 m(2 m-1)-3 m n+4 m(2 m-1)(s-2) \epsilon}{m s+4(2 m-1)-2 n+2(2 m-1)(s-2) \epsilon} .\end{cases}
\end{aligned}
$$

Then

$$
\|u\|_{L^{\infty}\left(\mathbb{B}_{1}\left(x_{0}\right)\right)} \geq c \exp \left[-C\left(n, m, s, \varepsilon, A_{0}, \cdots, A_{\alpha_{0}}\right) R^{\tilde{\Theta}} \log R\right] .
$$

Case III): For the case $2 \leq n<4 m-2$ and $s \in\left(\frac{4(2 m-1)}{3 m}, \infty\right]$. As before, we need to find the $\max \left\{(2 m-|\alpha|) \mu,\left(2 m-\frac{n}{s}\right) \bar{\nu}\right\}$. We can check that

$$
\begin{aligned}
& \max \left\{(2 m-|\alpha|) \mu,\left(2 m-\frac{n}{s}\right) \bar{\nu}\right\} \\
& =\bar{\Theta}:=\left\{\begin{array}{cc}
\frac{2\left(2 m-\alpha_{0}\right)}{3 m-2 \alpha_{0}} & \alpha_{0} \geq \frac{8 m(2 m-1)-3 m n}{m s+4(2 m-1)-2 n}, \\
\frac{2(2 m s-n)}{3 m s-4(2 m-1)} & \alpha_{0}<\frac{8 m(2 m-1)-3 m n}{m s+4(2 m-1)-2 n} .
\end{array}\right.
\end{aligned}
$$

Thus,

$$
\|u\|_{L^{\infty}\left(\mathbb{B}_{1}\left(x_{0}\right)\right)} \geq c \exp \left[-C\left(n, m, A_{0}, \cdots, A_{\alpha_{0}}\right) R^{\bar{\Theta}} \log R\right] .
$$

Therefore, the conclusion of the theorem follows.

\section{REFERENCES}

[ADN59] Shmuel Agmon, Avron Douglis and Louis Nirenberg. Estimates near the boundary for solutions of elliptic partial differential equations satisfying general boundary conditions I. Comm. Pure Appl. Math., 12:623-727, 1959.

[Bak12] Laurent Bakri. Quantitative uniqueness for Schrödinger operator. Indiana Univ. Math. J., 61(4):1565-1580, 2012.

[Bak13] Laurent Bakri. Carleman estimates for the Schrdinger Operator. Application to quantitative uniqueness. Communication in Partial Differential Equations, 38(1):69-91, 2013.

[BK05] Jean Bourgain and Carlos Kenig. On localization in the continuous Anderson-Bernoulli model in higher dimension. Invent. Math., 161(2):389-426, 2005.

[CG99] Ferruccio Colombini and Cataldo Grammatico. Some remarks on strong unique continuation for the Laplace operator and its powers. Comm. Partial Differential Equations, 24(5-6):1079-1094, 1999.

[CK10] Ferruccio Colombini and Herbert Koch. Strong unique continuation for products of elliptic operators of second order, Trans. Amer. Math. Soc., 362(1):345-355, 2010.

[Dav14] Blair Davey. Some quantitative unique continuation results for eigenfunctions of the magnetic Schrödinger operator. Comm. Partial Differential Equations, 39(5):876-945, 2014.

[DF88] Harold Donnelly and Charles Fefferman. Nodal sets of eigenfunctions on Riemannian manifolds. Invent. Math., 93(1):161-183, 1988. 
[DF90] Herold Donnelly and Charles Fefferman. Growth and geometry of eigenfunctions of the Laplacian. In Analysis and partial differential equations, volume 122 of Lecture Notes in Pure and Appl. Math., pages 635-655. Dekker, New York, 1990.

[DZ17] Blair Davey and Jiuyi Zhu. Quantitative uniqueness of solutions to second order elliptic equations with singular lower order terms, arXiv:1702.04742.

[DaZ17] Blair Davey and Jiuyi Zhu. Quantitative uniqueness of solutions to second order elliptic equations with singular potentials in two dimensions, arXiv:1704.00632.

[GL86] Nicola Garofalo and Fang-Hua Lin. Monotonicity properties of variational integrals, $A_{p}$ weights and unique continuation, Indiana Univ. Math., 35:245-268, 1986.

[GL87] Nicola Garofalo and Fang-Hua Lin. Unique continuation for elliptic operators: a geometric-variational approach, Comm. Pure Appl. Math., 40(3):347-366, 1987.

[H85] Lars Hörmander. The analysis of linear partial differential operators, Vol. 3, SpringerVerlag, Berlin, New York, 1985.

[HWZ16] Shanlin Huang, Ming Wang and Quan Zheng. Quantitative uniqueness of some higher order elliptic equations. J. Math. Anal. Appl., 444(1):326-339, 2016.

[JK85] David Jerison and Carlos Kenig. Unique continuation and absence of positive eigenvalues for Schrödinger operators. Ann. of Math., 121(3):463-494, 1985. With an appendix by E. M. Stein.

[Ken07] Carlos Kenig. Some recent applications of unique continuation. In Recent developments in nonlinear partial differential equations, volume 439 of Contemp. Math., pages 25-56. Amer. Math. Soc., Providence, RI, 2007.

[Ku98] Igor Kukavica. Quantitative uniqueness for second-order elliptic operators. Duke Math. J., 91(2):225-240, 1998.

[KSW15] Carlos Kenig, Luis Silvestre, and Jenn-Nan Wang. On Landis' conjecture in the plane. Comm. Partial Differential Equations, 40(4):766-789, 2015.

[KT01] Herbert Koch and Daniel Tataru. Carleman estimates and unique continuation for second-order elliptic equations with nonsmooth coefficients. Comm. Pure Appl. Math., 54(3):339-360, 2001.

[KT16] Abel Klein and C. S. Sidney Tsang. Quantitative unique continuation principle for Schrödinger operators with singular potentials. Proc. Amer. Math. Soc., 144(2):665-679, 2016.

[KW15] Carlos Kenig and Jenn-Nan Wang. Quantitative uniqueness estimates for second order elliptic equations with unbounded drift. Math. Res. Lett., 22(4):1159-1175, 2015.

[L07] Ching-Lung Lin. Strong unique continuation for $m$-th powers of a Laplacian operator with singular coefficients. Proc. Amer. Math. Soc., 135(2):569-578, 2007.

[LNW11] Ching-Lung Lin, Sei Nagayasu and Jenn-Nan Wang. Quantitative uniqueness for the power of the Laplacian with singular coefficients. Ann. Sc. Norm. Super. Pisa Cl. Sci., 10:513-529, 2011.

[LW14] Ching-Lung Lin and Jenn-Nan Wang. Quantitative uniqueness estimates for the general second order elliptic equations. J. Funct. Anal., 266(8):5108-5125, 2014.

[Mes92] V. Z. Meshkov. On the possible rate of decay at infinity of solutions of second order partial differential equations. Math USSR SB., 72:343-361, 1992.

[P60] Murray H Protter. unique continuation for elliptic equations, Trans. Amer. Math. Soc. 95:81-91, 1960.

[R97] Rachid Regbaoui. Strong uniqueness for second order differential operators, J. Diff. Eq., 141:201-217, 1997.

[S90] Christopher D. Sogge. Strong uniqueness theorems for second order elliptic differential equations. Amer. J. Math., 112:943-984, 1990.

[Wol92] Thomas H. Wolff. A property of measures in $\mathbb{R}^{N}$ and an application to unique continuation. Geom. Funct. Anal., 2(2): 225-284, 1992. 
[Zhu16] Jiuyi Zhu. Quantitative uniqueness of elliptic equations. Amer. J. Math., 138(3):733$762,2016$.

Department of Mathematics, Louisiana State University, Baton Rouge, LA 70803, USA, Email: ZHU@MATH.LSU.EDU 